\title{
Hadronic three-body $D$ decays mediated by scalar resonances
}

\author{
Hai-Yang Cheng $\odot,{ }^{1}$ Cheng-Wei Chiang $\odot,{ }^{2,3}$ and Zhi-Qing Zhang ${ }^{4}$ \\ ${ }^{1}$ Institute of Physics, Academia Sinica, Taipei, Taiwan 11529, Republic of China \\ ${ }^{2}$ Department of Physics, National Taiwan University, Taipei, Taiwan 10617, Republic of China \\ ${ }^{3}$ Physics Division, National Center for Theoretical Sciences, Taipei, Taiwan 10617, Republic of China \\ ${ }^{4}$ Department of Physics, Henan University of Technology, Zhengzhou, \\ Henan 450052, People's Republic of China
}

(Received 9 January 2022; accepted 27 January 2022; published 22 February 2022)

\begin{abstract}
We study the quasi-two-body $D \rightarrow S P$ decays and the three-body $D$ decays proceeding through intermediate scalar resonances, where $S$ and $P$ denote scalar and pseudoscalar mesons, respectively. Our main results are the following: (i) Certain external and internal $W$-emission diagrams with the emitted meson being a scalar meson are naïvely expected to vanish, but they actually receive contributions from vertex and hard spectator-scattering corrections beyond the factorization approximation. (ii) For light scalars with masses below or close to $1 \mathrm{GeV}$, it is more sensible to study three-body decays directly and compare with experiment as the two-body branching fractions are either unavailable or subject to large finite-width effects of the scalar meson. (iii) We consider the two-quark (scheme I) and four-quark (scheme II) descriptions of the light scalar mesons, and find the latter generally in better agreement with experiment. This is in line with recent BESIII measurements of semileptonic charm decays that prefer the tetraquark description of light scalars produced in charmed meson decays. (iv) The topological amplitude approach fails here as the $D \rightarrow S P$ decay branching fractions cannot be reliably inferred from the measurements of three-body decays, mainly because the decay rates cannot be factorized into the topological amplitude squared and the phase space factor. (v) The predicted rates for $D^{0} \rightarrow f_{0} P, a_{0} P$ are generally smaller than experimental data by one order of magnitude, presumably implying the significance of $W$-exchange amplitudes. (vi) The $W$-annihilation amplitude is found to be very sizable in the $S P$ sector with $|A / T|_{S P} \sim 1 / 2$, contrary to its suppression in the $P P$ sector with $|A / T|_{P P} \sim 0.18$. (vii) Finite-width effects are very important for the very broad $\sigma / f_{0}(500)$ and $\kappa / K_{0}^{*}(700)$ mesons. The experimental branching fractions $\mathcal{B}\left(D^{+} \rightarrow \sigma \pi^{+}\right)$and $\mathcal{B}\left(D^{+} \rightarrow \bar{\kappa}^{0} \pi^{+}\right)$are thus corrected to be $(3.8 \pm 0.3) \times 10^{-3}$ and $\left(6.7_{-4.5}^{+5.6}\right) \%$, respectively.
\end{abstract}

DOI: 10.1103/PhysRevD.105.033006

\section{INTRODUCTION}

In recent years many measurements of hadronic threebody and four-body decays of charmed mesons have been performed with Dalitz-plot amplitude analyses. Amplitudes describing $D$ meson decays into multibody final states are dominated by quasi-two-body processes, such as $D \rightarrow$ $P P, V P, S P, A P$ and $T P$, where $P, V, S, A$ and $T$ denote pseudoscalar, vector, scalar, axial-vector and tensor mesons, respectively. Among various $S$-, $P$ - and $D$-wave intermediate resonances, the identification of the scalar mesons is rather difficult due to their broad widths and flat angular distributions.

Published by the American Physical Society under the terms of the Creative Commons Attribution 4.0 International license. Further distribution of this work must maintain attribution to the author(s) and the published article's title, journal citation, and DOI. Funded by SCOAP ${ }^{3}$.
Scalar mesons with masses lower than $2 \mathrm{GeV}$ can be classified into two nonets: one nonet with masses below or close to $1 \mathrm{GeV}$, including $\sigma / f_{0}(500), f_{0}(980), a_{0}(980)$ and $\kappa / K_{0}^{*}(700)$; and the other nonet with masses above $1 \mathrm{GeV}$, including $a_{0}(1450), K_{0}^{*}(1430), f_{0}(1370), f_{0}(1500)$ and $f_{0}(1710)$. The last three are all isosinglet scalars and only two of them can be accommodated in the quark model, implying a dominant scalar glueball content in one of the three isosinglets.

In this work, we shall study the quasi-two-body $D \rightarrow S P$ decays and the three-body $D$ decays proceeding through intermediate scalar resonances. In Tables I and II we collect all the measured branching fractions of $D \rightarrow S P \rightarrow P_{1} P_{2} P$ decays available in the Particle Data Group (PDG) [1]. It is clear that $f_{0}(980)$ and the $f_{0}$ family such as $f_{0}(1370)$, $f_{0}(1500)$ and $f_{0}(1710)$ are observed in the three-body decays of $D^{+}, D^{0}$ and $D_{s}^{+}$, while $a_{0}(980)$ is seen exclusively in three-body $D^{0}$ decays (except for $D_{s}^{+} \rightarrow a_{0}^{+, 0} \pi^{0,+}$ ). Contrary to $f_{0}(980)$ and $a_{0}(980)$ which are relatively easy to identify experimentally, the establishment of $\sigma$ and $\kappa$ is 
very difficult and controversial because their widths are so broad that their shapes are not clearly resonant. Nevertheless, their signals in three-body $D$ decays have been identified in $D^{+, 0} \rightarrow \sigma \pi^{+, 0} \rightarrow \pi^{+} \pi^{-} \pi^{+, 0}, D^{+} \rightarrow$ $\bar{\kappa}^{0} \pi^{+} \rightarrow K_{S} \pi^{0} \pi^{+}$and $D^{+} \rightarrow \bar{\kappa}^{0} K^{+} \rightarrow \pi^{+} K^{-} K^{+}$, respectively. Because of threshold and coupled-channel effects for $f_{0}(980)$ and $a_{0}(980)$ and the very broad widths for $\sigma$ and $\kappa$, it is no longer pertinent to use the conventional BreitWigner parametrization to describe their line shapes.

The $D \rightarrow S P$ decays and related three-body $D$ decays have been studied previously in Refs. [7-18]. In the $D \rightarrow$ $S P$ decays, the flavor diagram of each topology has two possibilities: one with the spectator quark in the charmed meson going to the pseudoscalar meson in the final state, and the other with the spectator quark ending up in the scalar meson. We thus need two copies of each topological diagram to describe the decay processes. Many of these decays have been observed in recent years through dedicated experiments and powerful Dalitz plot analyses of multibody decays. We will investigate whether an extraction of the sizes and relative strong phases of these amplitudes is possible.

One purpose of studying these decays is to check our understanding in the structures and properties of light evenparity scalar mesons. Another goal is to learn the final-state interaction pattern in view of the rich resonance spectrum around the $D$ meson mass range. Not only does this work update our previous study [14], we also study the finite-width effect in the three-body decays mediated by the scalar mesons. Such an effect is observed to be particularly important for decays involving $\sigma / f_{0}(500)$ and $\kappa / K_{0}^{*}(700)$ in the intermediate state because of their broad widths compared to their masses, respectively. Therefore, one should be careful in the use of the narrow width approximation (NWA) to extract the $D \rightarrow S P$ twobody decays from the three-body decay rates.

This paper is organized as follows. In Sec. II, we review the current experimental status about how various $D \rightarrow S P$ decay branching fractions are extracted using the NWA from three-body decay rates. In Sec. III, we discuss the two-quark $q \bar{q}$ and tetraquark pictures of the scalar nonet near or below $1 \mathrm{GeV}$ along with the associated conundrums. The decay constants and form factors required for subsequent numerical calculations are given in this section, too. Section IV sets up the notation and formalism of flavor amplitude analysis, for both quark-antiquark and tetraquark pictures. In Sec. V, we take the factorization approach as an alternative toward analyzing these decays. We also introduce line shapes for the scalar resonances when describing various three-body decays. Section VI gives the results obtained based upon the approaches in the previous two sections for a comparison. Section VIB is devoted to the study of finite-width effect and how the NWA should be modified. We summarize our findings in Sec. VII.

\section{EXPERIMENTAL STATUS}

It is known that three- and four-body decays of heavy mesons provide a rich laboratory for studying the intermediate-state resonances. The Dalitz plot analysis of threebody or four-body decays of charmed mesons is a very useful technique for this purpose. We are interested in $D \rightarrow S P$ decays followed by $S \rightarrow P_{1} P_{2}$. The results of various experiments are summarized in Tables I and II. To extract the branching fraction for a $D \rightarrow S P$ decay, it is the usual practice to use the NWA:

$\Gamma\left(D \rightarrow S P \rightarrow P_{1} P_{2} P\right)=\Gamma(D \rightarrow S P)_{\mathrm{NWA}} \mathcal{B}\left(S \rightarrow P_{1} P_{2}\right)$.

Since this relation holds only in the $\Gamma_{S} \rightarrow 0$ limit, we put the subscript NWA to emphasize that $\mathcal{B}(D \rightarrow S P)$ thus obtained is under this limit. Finite width effects will be discussed in Sec. VI B. For the branching fractions of twobody decays of scalar mesons, we shall use [1]

$$
\begin{aligned}
\mathcal{B}\left(a_{0}(980) \rightarrow \pi \eta\right) & =0.850 \pm 0.017, \\
\mathcal{B}\left(\sigma(500) \rightarrow \pi^{+} \pi^{-}\right) & =\frac{2}{3}, \\
\mathcal{B}\left(f_{0}(1500) \rightarrow \pi \pi\right) & =0.345 \pm 0.022, \\
\mathcal{B}\left(f_{0}(1710) \rightarrow K^{+} K^{-}\right) & =0.292 \pm 0.027, \\
\mathcal{B}\left(K_{0}^{* 0}(1430) \rightarrow K^{+} \pi^{-}\right) & =\frac{2}{3}(0.93 \pm 0.10), \\
\mathcal{B}\left(\kappa(700) \rightarrow K^{+} \pi^{-}\right) & =\frac{2}{3},
\end{aligned}
$$

where we have applied the average of $\Gamma\left(a_{0}(980) \rightarrow\right.$ $K \bar{K}) / \Gamma\left(a_{0}(980) \rightarrow \pi \eta\right)=0.177 \pm 0.024$ from PDG [1] to extract the branching fraction of $a_{0}(980) \rightarrow \pi \eta$, assuming that its width is saturated by the $K \bar{K}$ and $\pi \eta$ modes. For $f_{0}(1710)$ we have used the values of $\Gamma\left(f_{0}(1710) \rightarrow \pi \pi\right) / \Gamma\left(f_{0}(1710) \rightarrow K \bar{K}\right)=0.23 \pm 0.05$ and $\Gamma\left(f_{0}(1710) \rightarrow \eta \eta\right) / \Gamma\left(f_{0}(1710) \rightarrow K \bar{K}\right)=0.48 \pm 0.15$ from PDG together with the assumption of its width being saturated by $\pi \pi, K \bar{K}$ and $\eta \eta$ modes. For $S=f_{0}(980)$ or $a_{0}(980)$, we are not able to extract the branching fractions of $D \rightarrow S P$ due to the lack of information of $\mathcal{B}\left(S \rightarrow P_{1} P_{2}\right)$ [except for $a_{0}(980) \rightarrow \pi \eta$ ], especially for $\mathcal{B}(S \rightarrow K \bar{K})$ where the threshold effect must be taken into account. For example, the NWA relation

$$
\begin{aligned}
& \Gamma\left(D^{+} \rightarrow f_{0}(980) K^{+} \rightarrow K^{+} K^{-} K^{+}\right) \\
& \quad=\Gamma\left(D^{+} \rightarrow f_{0}(980) K^{+}\right) \mathcal{B}\left(f_{0}(980) \rightarrow K^{+} K^{-}\right)
\end{aligned}
$$

cannot be applied to extract the branching fraction of $D^{+} \rightarrow$ $f_{0}(980) K^{+}$due to the unknown $\mathcal{B}\left(f_{0}(980) \rightarrow K^{+} K^{-}\right)$. Therefore, we will calculate the branching fractions of 
TABLE I. Experimental branching fractions of $\left(D^{+}, D_{s}^{+}\right) \rightarrow S P \rightarrow P_{1} P_{2} P$ decays. For simplicity and convenience, we have dropped the mass identification for $\sigma(500), f_{0}(980), a_{0}(980), \kappa(700)$ and $K_{0}^{*}(1430)$. Data are taken from Ref. [1] unless specified otherwise. We have applied the NWA given by Eq. (2.1) to extract the branching fractions of the two-body $D$ decay denoted by $\mathcal{B}(D \rightarrow S P)_{\mathrm{NWA}}$.

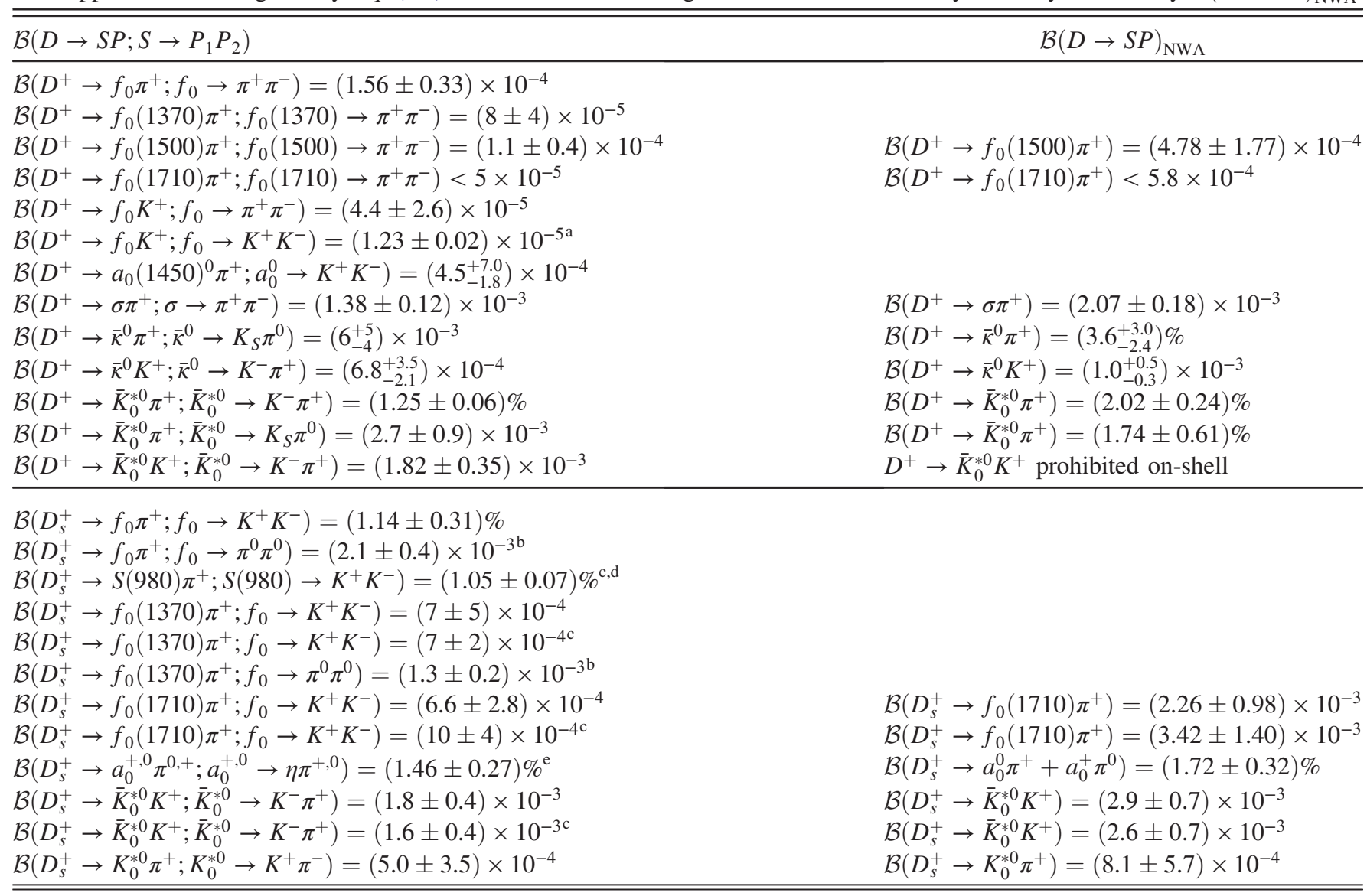

${ }^{a}$ Assuming a fit fraction of $20 \%$ for $D^{+} \rightarrow f_{0}(980) K^{+}$in $D^{+} \rightarrow K^{+} K^{-} K^{+}$decay [2].

${ }^{\mathrm{b}}$ BESIII data taken from Ref. [3].

${ }^{\mathrm{c}}$ BESIII data taken from Ref. [4].

${ }^{\mathrm{d}} S(980)$ denotes both $f_{0}(980)$ and $a_{0}(980)$.

${ }^{\mathrm{e}}$ The branching fraction is assigned to be $(2.2 \pm 0.4) \%$ by the PDG [1]. However, as pointed out in Ref. [5], the fraction of $D_{s}^{+} \rightarrow a_{0}(980)^{+(0)} \pi^{0(+)}, a_{0}(980)^{+(0)} \rightarrow \pi^{0(+)} \eta$ with respect to the total fraction of $D_{s}^{+} \rightarrow a_{0}(980) \pi, a_{0}(980) \rightarrow \pi \eta$ is evaluated to be 0.66 . Consequently, the branching fraction should be multiplied by a factor of 0.66 to become $(1.46 \pm 0.27) \%$.

$\mathcal{B}\left(D \rightarrow S P \rightarrow P_{1} P_{2} P\right)$ directly and compare them with experiment (see Table VIII below).

\section{PHYSICAL PROPERTIES OF SCALAR MESONS}

It is known that the underlying structure of scalar mesons is not well established theoretically (see, e.g., Refs. [19,20] for a review). Scalar mesons with masses lower than $2 \mathrm{GeV}$ can be classified into two nonets: one nonet with masses below or close to $1 \mathrm{GeV}$, including the isoscalars $f_{0}(500)$ (or $\sigma$ ), $f_{0}(980)$, the isodoublet $K_{0}^{*}(700)$ (or $\kappa$ ) and the isovector $a_{0}(980)$; and the other nonet with masses above $1 \mathrm{GeV}$, including $f_{0}(1370), a_{0}(1450), K_{0}^{*}(1430)$ and $f_{0}(1500) / f_{0}(1710)$. If the scalar meson states below or near $1 \mathrm{GeV}$ are identified as the conventional low-lying $0^{+} q \bar{q}$ nonet, then the nonet states above $1 \mathrm{GeV}$ could be excited $q \bar{q}$ states.
In the naïve quark model, the flavor wave functions of the light scalars read

$$
\begin{aligned}
\sigma & =\frac{1}{\sqrt{2}}(u \bar{u}+d \bar{d}), & f_{0} & =s \bar{s}, \\
a_{0}^{0} & =\frac{1}{\sqrt{2}}(u \bar{u}-d \bar{d}), & a_{0}^{+}=u \bar{d}, & \\
\kappa^{+} & =u \bar{s}, \quad \kappa^{0}=d \bar{s}, & \bar{\kappa}^{0}=s \bar{d}, & \kappa^{-}=s \bar{u},
\end{aligned}
$$

where an ideal mixing for $f_{0}$ and $\sigma$ is assumed as $f_{0}(980)$ is the heaviest one and $\sigma$ the lightest one in the light scalar nonet. However, as summarized in Ref. [14], this simple picture encounters several serious problems:

(1) It is impossible to understand the mass degeneracy between $f_{0}(980)$ and $a_{0}(980)$, which is the so-called "inverted spectrum problem." 
TABLE II. Same as Table I except for $D^{0} \rightarrow S P \rightarrow P_{1} P_{2} P$ decays.

\begin{tabular}{|c|c|}
\hline $\mathcal{B}\left(D \rightarrow S P ; S \rightarrow P_{1} P_{2}\right)$ & $\mathcal{B}(D \rightarrow S P)_{\mathrm{NWA}}$ \\
\hline \\
\hline \multicolumn{2}{|l|}{$\mathcal{B}\left(D^{0} \rightarrow f_{0} \pi^{0} ; f_{0} \rightarrow K^{+} K^{-}\right)=(3.6 \pm 0.6) \times 10^{-4}$} \\
\hline \multicolumn{2}{|l|}{$\mathcal{B}\left(D^{0} \rightarrow f_{0}(1370) \pi^{0} ; f_{0} \rightarrow \pi^{+} \pi^{-}\right)=(5.5 \pm 2.1) \times 10^{-5}$} \\
\hline $\mathcal{B}\left(D^{0} \rightarrow f_{0}(1500) \pi^{0} ; f_{0} \rightarrow \pi^{+} \pi^{-}\right)=(5.8 \pm 1.6) \times 10^{-5}$ & $\mathcal{B}\left(D^{0} \rightarrow f_{0}(1500) \pi^{0}\right)=(2.5 \pm 0.7) \times 10^{-4}$ \\
\hline $\mathcal{B}\left(D^{0} \rightarrow f_{0}(1710) \pi^{0} ; f_{0} \rightarrow \pi^{+} \pi^{-}\right)=(4.6 \pm 1.6) \times 10^{-5}$ & $\mathcal{B}\left(D^{0} \rightarrow f_{0}(1710) \pi^{0}\right)=(3.7 \pm 1.4) \times 10^{-4}$ \\
\hline \multicolumn{2}{|l|}{$\mathcal{B}\left(D^{0} \rightarrow f_{0} \bar{K}^{0} ; f_{0} \rightarrow \pi^{+} \pi^{-}\right)=\left(2.40_{-046}^{+0.80}\right) \times 10^{-3}$} \\
\hline \multicolumn{2}{|l|}{$\mathcal{B}\left(D^{0} \rightarrow f_{0} \bar{K}^{0} ; f_{0} \rightarrow K^{+} K^{-}\right)<1.8 \times 10^{-4}$} \\
\hline \multicolumn{2}{|l|}{$\mathcal{B}\left(D^{0} \rightarrow f_{0}(1370) \bar{K}^{0} ; f_{0} \rightarrow \pi^{+} \pi^{-}\right)=\left(5.6_{-2.6}^{+1.8}\right) \times 10^{-3}$} \\
\hline \multicolumn{2}{|l|}{$\mathcal{B}\left(D^{0} \rightarrow f_{0}(1370) \bar{K}^{0} ; f_{0} \rightarrow K^{+} K^{-}\right)=(3.4 \pm 2.2) \times 10^{-4}$} \\
\hline \multicolumn{2}{|l|}{$\mathcal{B}\left(D^{0} \rightarrow a_{0}^{+} K^{-} ; a_{0}^{+} \rightarrow K^{+} \bar{K}^{0}\right)=(1.18 \pm 0.36) \times 10^{-3}$} \\
\hline \multicolumn{2}{|l|}{$\mathcal{B}\left(D^{0} \rightarrow a_{0}^{+} K^{-} ; a_{0}^{+} \rightarrow K^{+} \bar{K}^{0}\right)=(3.07 \pm 0.84) \times 10^{-3 \mathrm{a}}$} \\
\hline \multicolumn{2}{|l|}{$\mathcal{B}\left(D^{0} \rightarrow a_{0}^{-} K^{+} ; a_{0}^{-} \rightarrow K^{-} \bar{K}^{0}\right)<2.2 \times 10^{-4}$} \\
\hline \multicolumn{2}{|l|}{$\mathcal{B}\left(D^{0} \rightarrow a_{0}^{0} \bar{K}^{0} ; a_{0}^{0} \rightarrow K^{+} K^{-}\right)=(5.8 \pm 0.8) \times 10^{-3}$} \\
\hline \multicolumn{2}{|l|}{$\mathcal{B}\left(D^{0} \rightarrow a_{0}^{0} \bar{K}^{0} ; a_{0}^{0} \rightarrow K^{+} K^{-}\right)=(8.12 \pm 1.80) \times 10^{-3 \mathrm{a}}$} \\
\hline $\mathcal{B}\left(D^{0} \rightarrow a_{0}^{0} \bar{K}^{0} ; a_{0}^{0} \rightarrow \eta \pi^{0}\right)=(2.40 \pm 0.56) \times 10^{-2}$ & $\mathcal{B}\left(D^{0} \rightarrow a_{0}^{0} \bar{K}^{0}\right)=(2.83 \pm 0.66) \%$ \\
\hline \multicolumn{2}{|l|}{$\mathcal{B}\left(D^{0} \rightarrow a_{0}^{-} \pi^{+} ; a_{0}^{-} \rightarrow K^{-} K^{0}\right)=(2.6 \pm 2.8) \times 10^{-4}$} \\
\hline \multicolumn{2}{|l|}{$\mathcal{B}\left(D^{0} \rightarrow a_{0}^{+} \pi^{-} ; a_{0}^{+} \rightarrow K^{+} \bar{K}^{0}\right)=(1.2 \pm 0.8) \times 10^{-3}$} \\
\hline \multicolumn{2}{|l|}{$\mathcal{B}\left(D^{0} \rightarrow a_{0}(1450)^{-} \pi^{+} ; a_{0}^{-} \rightarrow K^{-} K^{0}\right)=(5.0 \pm 4.0) \times 10^{-5}$} \\
\hline \multicolumn{2}{|l|}{$\mathcal{B}\left(D^{0} \rightarrow a_{0}(1450)^{+} \pi^{-} ; a_{0}^{+} \rightarrow K^{+} \bar{K}^{0}\right)=(6.4 \pm 5.0) \times 10^{-5}$} \\
\hline \multicolumn{2}{|l|}{$\mathcal{B}\left(D^{0} \rightarrow a_{0}(1450)^{-} K^{+} ; a_{0}^{-} \rightarrow K^{-} K_{S}\right)<0.6 \times 10^{-3 \mathrm{a}}$} \\
\hline $\mathcal{B}\left(D^{0} \rightarrow \sigma \pi^{0} ; \sigma \rightarrow \pi^{+} \pi^{-}\right)=(1.22 \pm 0.22) \times 10^{-4}$ & $\mathcal{B}\left(D^{0} \rightarrow \sigma \pi^{0}\right)=(1.8 \pm 0.3) \times 10^{-4}$ \\
\hline $\mathcal{B}\left(D^{0} \rightarrow K_{0}^{*-} \pi^{+} ; K_{0}^{*-} \rightarrow \bar{K}^{0} \pi^{-}\right)=\left(5.34_{-0.66}^{+0.80}\right) \times 10^{-3}$ & $\mathcal{B}\left(D^{0} \rightarrow K_{0}^{*-} \pi^{+}\right)=\left(8.6_{-1.4}^{+1.6}\right) \times 10^{-3}$ \\
\hline $\mathcal{B}\left(D^{0} \rightarrow K_{0}^{*-} \pi^{+} ; K_{0}^{*-} \rightarrow K^{-} \pi^{0}\right)=(4.8 \pm 2.2) \times 10^{-3}$ & $\mathcal{B}\left(D^{0} \rightarrow K_{0}^{*-} \pi^{+}\right)=(1.55 \pm 0.73) \%$ \\
\hline $\mathcal{B}\left(D^{0} \rightarrow \bar{K}_{0}^{* 0} \pi^{0} ; \bar{K}_{0}^{* 0} \rightarrow K^{-} \pi^{+}\right)=\left(5.9_{-1.6}^{+5.0}\right) \times 10^{-3}$ & $\mathcal{B}\left(D^{0} \rightarrow \bar{K}_{0}^{* 0} \pi^{0}\right)=\left(9.5_{-2.8}^{+8.1}\right) \times 10^{-3}$ \\
\hline $\mathcal{B}\left(D^{0} \rightarrow K_{0}^{*+} \pi^{-} ; K_{0}^{*+} \rightarrow K^{0} \pi^{+}\right)<2.8 \times 10^{-5}$ & $\mathcal{B}\left(D^{0} \rightarrow K_{0}^{*+} \pi^{-}\right)<4.5 \times 10^{-5}$ \\
\hline
\end{tabular}

${ }^{\mathrm{a} B E S I I I}$ data taken from Ref. [6].

(2) The $P$-wave $0^{+}$meson has one unit of orbital angular momentum which costs an energy around $500 \mathrm{MeV}$. Hence, it should have a mass lying above rather than below $1 \mathrm{GeV}$.

(3) It is difficult to explain why $\sigma$ and $\kappa$ are much broader than $f_{0}(980)$ and $a_{0}(980)$ in width.

(4) The $\gamma \gamma$ widths of $a_{0}(980)$ and $f_{0}(980)$ are much smaller than naïvely expected for a $q \bar{q}$ state [21].

(5) The radiative decay $\phi \rightarrow a_{0}(980) \gamma$, which cannot proceed if $a_{0}(980)$ is a pure $q \bar{q}$ state, can be nicely described by the four-quark nature of $a_{0}(980)$ [22,23] or the kaon loop mechanism [24]. Likewise, the observation of the radiative decay $\phi \rightarrow$ $f_{0}(980) \gamma \rightarrow \pi \pi \gamma$ is also accounted for by the four-quark state of $f_{0}(980)$ [23].

It turns out that these difficulties can be readily resolved in the tetraquark scenario where the four-quark flavor wave functions of light scalar mesons are symbolically given by [25]

$$
\begin{aligned}
\sigma & =u \bar{u} d \bar{d}, \quad f_{0}=\frac{1}{\sqrt{2}}(u \bar{u}+d \bar{d}) s \bar{s}, \\
a_{0}^{0} & =\frac{1}{\sqrt{2}}(u \bar{u}-d \bar{d}) s \bar{s}, \quad a_{0}^{+}=u \bar{d} s \bar{s}, \quad a_{0}^{-}=d \bar{u} s \bar{s}, \\
\kappa^{+} & =u \bar{s} d \bar{d}, \quad \kappa^{0}=d \bar{s} u \bar{u}, \quad \bar{\kappa}^{0}=s \bar{d} u \bar{u}, \quad \kappa^{-}=s \bar{u} d \bar{d} .
\end{aligned}
$$

The four quarks $q^{2} \bar{q}^{2}$ can form an $S$-wave (rather than $P$-wave) $0^{+}$meson without introducing one unit of orbital angular momentum. This four-quark description explains naturally the inverted mass spectrum of the light nonet, ${ }^{1}$ especially the mass degeneracy between $f_{0}(980)$ and $a_{0}(980)$, and accounts for the broad widths of $\sigma$ and $\kappa$ while $f_{0}(980)$ and $a_{0}(980)$ are narrow because of the suppressed phase space for their decays to the kaon pairs. Lattice calculations have confirmed that $a_{0}(1450)$ and $K_{0}^{*}(1430)$ are $q \bar{q}$ mesons, and suggested that $\sigma, \kappa$ and $a_{0}(980)$ are tetraquark mesonia [27-31].

The inverted spectrum problem can also be alleviated in the scenario where the light scalars are dynamically generated from the meson-meson interaction, with the $f_{0}(980)$ and the $a_{0}(980)$ coupling strongly to the $K \bar{K}$ channel with isospin 0 and 1 , respectively. Indeed, the whole light scalar nonet appears naturally from properly

\footnotetext{
${ }^{1}$ However, it has been claimed recently in Ref. [26] that the inverse mass hierarchy can be realized in the $q \bar{q}$ picture through a $U(1)$ axial anomaly including explicit $S U(3)_{F}$ breaking. The anomaly term contributes to $a_{0}(980)$ with the strange quark mass and to $\kappa / K_{0}^{*}(700)$ with the up or down quark mass due to its flavor singlet nature. The current mass of the strange quark makes the $a_{0}$ meson heavier than the $\kappa$ meson.
} 
unitarized chiral amplitudes for pseudoscalar-pseudoscalar scatterings [32,33]. Consequently, both $f_{0}(980)$ and $a_{0}(980)$ are good candidates of $K \bar{K}$ molecular states [34], while $\sigma$ and $\kappa$ can be considered as the bound states of $\pi \pi$ and $K \pi$, respectively.

In the naïve two-quark model with ideal mixing for $f_{0}(980)$ and $\sigma(500), f_{0}(980)$ is purely an $s \bar{s}$ state, while $\sigma(500)$ is an $n \bar{n}$ state with $n \bar{n} \equiv(\bar{u} u+\bar{d} d) / \sqrt{2}$. However, there also exists some experimental evidence indicating that $f_{0}(980)$ is not a purely $s \bar{s}$ state. For example, the observation of $\Gamma\left(J / \psi \rightarrow f_{0} \omega\right) \approx \frac{1}{2} \Gamma\left(J / \psi \rightarrow f_{0} \phi\right)$ [1] clearly shows the existence of the nonstrange and strange quark contents in $f_{0}(980)$. Therefore, isoscalars $\sigma(500)$ and $f_{0}(980)$ must have a mixing

$$
\begin{aligned}
\left|f_{0}(980)\right\rangle & =|s \bar{s}\rangle \cos \theta+|n \bar{n}\rangle \sin \theta, \\
|\sigma(500)\rangle & =-|s \bar{s}\rangle \sin \theta+|n \bar{n}\rangle \cos \theta
\end{aligned}
$$

Various mixing angle measurements have been discussed in the literature and summarized in Refs. [35,36]. A recent measurement of the upper limit on the branching fraction product $\mathcal{B}\left(\bar{B}^{0} \rightarrow J / \psi f_{0}(980)\right) \times \mathcal{B}\left(f_{0}(980) \rightarrow \pi^{+} \pi^{-}\right)$by LHCb leads to $|\theta|<30^{\circ}$ [37]. Likewise, in the four-quark scenario for light scalar mesons, one can also define a similar $f_{0}-\sigma$ mixing angle

$$
\begin{aligned}
\left|f_{0}(980)\right\rangle & =|n \bar{n} s \bar{s}\rangle \cos \phi+|u \bar{u} d \bar{d}\rangle \sin \phi, \\
|\sigma(500)\rangle & =-|n \bar{n} s \bar{s}\rangle \sin \phi+|u \bar{u} d \bar{d}\rangle \cos \phi .
\end{aligned}
$$

It has been shown that $\phi=174.6^{\circ}$ [38].

In reality, the light scalar mesons could have both twoquark and four-quark components. Indeed, a real hadron in the QCD language should be described by a set of Fock states each of which has the same quantum number as the hadron. For example,

$$
\begin{aligned}
\left|a^{+}(980)\right\rangle= & \psi_{u \bar{d}}^{a_{0}}|u \bar{d}\rangle+\psi_{u \bar{d} g}^{a_{0}}|u \bar{d} g\rangle \\
& +\psi_{u \bar{u} s \bar{s}}^{a_{0}}|u \bar{d} s \bar{s}\rangle+\ldots
\end{aligned}
$$

In the tetraquark model, $\psi_{u \bar{d} s \bar{s}}^{a_{0}} \gg \psi_{u \bar{d}}^{a_{0}}$, while it is the other way around in the two-quark model. Although as far as the spectrum and decay are concerned, light scalars are predominately tetraquark states, their productions in heavy meson decays and in high energy hadron collisions are probably more sensitive to the two-quark component of the scalar mesons. For example, one may wonder if the energetic $f_{0}(980)$ produced in $B$ decays is dominated by the fourquark configuration as it requires to pick up two energetic quark-antiquark pairs to form a fast moving light tetraquark. Since the scalar meson production in charm decays is not energetic, it is possible that it has adequate time to form a tetraquark state. In principle, the two-quark and four-quark descriptions of the light scalars can be discriminated in the semileptonic charm decays. For example, the ratio

$$
R=\frac{\mathcal{B}\left(D^{+} \rightarrow f_{0} \ell^{+} \nu\right)+\mathcal{B}\left(D^{+} \rightarrow \sigma \ell^{+} \nu\right)}{\mathcal{B}\left(D^{+} \rightarrow a_{0}^{0} \ell^{+} \nu\right)}
$$

is equal to 1 in the two-quark scenario and 3 in the fourquark model under the flavor SU(3) symmetry [39]. Based on the BESIII measurements of $D^{+} \rightarrow a_{0}(980)^{0} e^{+} \nu_{e}$ [40], $D^{+} \rightarrow \sigma e^{+} \nu_{e}$ and the upper limit on $D^{+} \rightarrow f_{0}(980) e^{+} \nu_{e}$ [41], it follows that $R>2.7$ at $90 \%$ confidence level. Hence, the BESIII results favor the SU(3) nonet tetraquark description of the $f_{0}(500), f_{0}(980)$ and $a_{0}(980)$ produced in charmed meson decays. A detailed analysis of BESIII and CLEO data on the decays $D^{+} \rightarrow \pi^{+} \pi^{-} e^{+} \nu_{e}$ and $D_{s}^{+} \rightarrow \pi^{+} \pi^{-} e^{+} \nu_{e}$ in Ref. [42] also shows results in favor of the four-quark nature of light scalar mesons $f_{0}(500)$ and $f_{0}(980)$.

The vector and scalar decay constants of the scalar meson are, respectively, defined as

$$
\left\langle S(p)\left|\bar{q}_{2} \gamma_{\mu} q_{1}\right| 0\right\rangle=f_{S} p_{\mu}, \quad\left\langle S\left|\bar{q}_{2} q_{1}\right| 0\right\rangle=m_{S} \bar{f}_{S} .
$$

The neutral scalar mesons $\sigma, f_{0}$ and $a_{0}^{0}$ cannot be produced via the vector current owing to charge conjugation invariance or conservation of vector current:

$$
f_{\sigma}=f_{f_{0}}=f_{a_{0}^{0}}=0 .
$$

Applying the equation of motion to Eq. (3.7) yields

$$
\mu_{S} f_{S}=\bar{f}_{S}, \quad \text { with } \quad \mu_{S}=\frac{m_{S}}{m_{2}(\mu)-m_{1}(\mu)},
$$

where $m_{2}$ and $m_{1}$ are the running current quark masses. Therefore, the vector decay constant of the scalar meson $f_{S}$ vanishes in the $\mathrm{SU}(3)$ or isospin limit. The vector decay constants of $K_{0}^{*}(1430)$ and the charged $a_{0}(980)$ are nonvanishing, but they are suppressed due to the small mass difference between the constituent $s$ and $u$ quarks and between $d$ and $u$ quarks, respectively. The scalar decay constants $\bar{f}_{S}$ have been computed in Ref. [35] within the framework of QCD sum rules. For the reader's convenience, we list the scalar decay constants (in units of $\mathrm{MeV}$ ) at $\mu=1 \mathrm{GeV}$ relevant to the present work

$$
\begin{array}{rlrl}
\bar{f}_{f_{0}} & =370 \pm 20, & & \bar{f}_{a_{0}}=365 \pm 20, \\
\bar{f}_{\sigma} & =350 \pm 20, & & \bar{f}_{\kappa}=340 \pm 20, \\
\bar{f}_{a_{0}(1450)} & =460 \pm 50, & f_{f_{0}(1500)}=490 \pm 50, \\
\bar{f}_{K_{0}^{*}} & =445 \pm 50 . &
\end{array}
$$

From Eq. (3.9) we obtain (in units of MeV) ${ }^{2}$

\footnotetext{
${ }^{2}$ The vector decay constants of the scalar meson and its antiparticle are of opposite sign. For example, $f_{a_{0}(980)^{+}}=$ $-1.3 \mathrm{MeV}$ and $f_{a_{0}(980)^{-}}=1.3 \mathrm{MeV}$.
} 


$$
\left|f_{a_{0}(980)^{ \pm}}\right|=1.3, \quad\left|f_{a_{0}(1450)^{ \pm}}\right|=1.1, \quad\left|f_{\kappa}\right|=45.5, \quad\left|f_{K_{0}^{*}(1430)}\right|=35.3 .
$$

In short, the vector decay constants of scalar mesons are either zero or very small for nonstrange scalar mesons.

Form factors for $D \rightarrow P, S$ transitions are defined by [43]

$$
\begin{aligned}
& \left\langle P\left(p^{\prime}\right)\left|V_{\mu}\right| D(p)\right\rangle=\left(P_{\mu}-\frac{m_{D}^{2}-m_{P}^{2}}{q^{2}} q_{\mu}\right) F_{1}^{D P}\left(q^{2}\right)+\frac{m_{D}^{2}-m_{P}^{2}}{q^{2}} q_{\mu} F_{0}^{D P}\left(q^{2}\right), \\
& \left\langle S\left(p^{\prime}\right)\left|A_{\mu}\right| D(p)\right\rangle=-i\left[\left(P_{\mu}-\frac{m_{D}^{2}-m_{S}^{2}}{q^{2}} q_{\mu}\right) F_{1}^{D S}\left(q^{2}\right)+\frac{m_{D}^{2}-m_{S}^{2}}{q^{2}} q_{\mu} F_{0}^{D S}\left(q^{2}\right)\right],
\end{aligned}
$$

where $P_{\mu}=\left(p+p^{\prime}\right)_{\mu}$ and $q_{\mu}=\left(p-p^{\prime}\right)_{\mu}$. As shown in Ref. [44], a factor of $(-i)$ is needed in the $D \rightarrow S$ transition in order for the $D \rightarrow S$ form factors to be positive. This can also be checked from heavy quark symmetry consideration [44].

Throughout this paper, we use the 3-parameter parametrization

$$
F\left(q^{2}\right)=\frac{F(0)}{1-a\left(q^{2} / m_{D}^{2}\right)+b\left(q^{2} / m_{D}^{2}\right)^{2}}
$$

for $D \rightarrow S$ transitions. For hadronic $D \rightarrow S P$ decays, the relevant form factor is $F_{0}^{D S}\left(q^{2}\right)$. The parameters $F_{0}^{D S}(0)$ for $D \rightarrow S$ transitions calculated in the covariant light-front quark model (CLFQM) [44,45], covariant confined quark model (CCQM) [46], and light-cone sum rules (LCSR) [47-49] are exhibited in Table III. Note that the matrix element $\left\langle S\left(p^{\prime}\right)\left|A_{\mu}\right| D(p)\right\rangle$ is sometimes parametrized as

$$
\left\langle S\left(p^{\prime}\right)\left|A_{\mu}\right| D(p)\right\rangle=-i\left[F_{+}^{D S}\left(q^{2}\right) P_{\mu}+F_{-}^{D S}\left(q^{2}\right) q_{\mu}\right] .
$$

It is easily seen that

$$
\begin{aligned}
& F_{1}\left(q^{2}\right)=F_{+}\left(q^{2}\right), \\
& F_{0}\left(q^{2}\right)=\frac{q^{2}}{m_{D}^{2}-m_{S}^{2}} F_{-}\left(q^{2}\right)+F_{+}\left(q^{2}\right),
\end{aligned}
$$

and hence $F_{1}(0)=F_{0}(0)=F_{+}(0)$. It was argued in [49] that the relation $F_{-}\left(q^{2}\right)=-F_{+}\left(q^{2}\right)$ holds in the LCSR calculation. In [46], the $D \rightarrow S$ transition form factors are defined by

$$
\left\langle S(p)\left|A_{\mu}\right| D(p+q)\right\rangle=-i\left[F_{+}^{\prime}\left(q^{2}\right) p_{\mu}+F_{-}^{\prime}\left(q^{2}\right) q_{\mu}\right] .
$$

They are related to $F_{+}\left(q^{2}\right)$ and $F_{-}\left(q^{2}\right)$ through the relation

$$
\begin{aligned}
& F_{+}^{\prime}\left(q^{2}\right)=2 F_{+}\left(q^{2}\right), \\
& F_{-}^{\prime}\left(q^{2}\right)=F_{+}\left(q^{2}\right)+F_{-}\left(q^{2}\right) .
\end{aligned}
$$

For the $q^{2}$ dependence of the form factors in various models, the parameters $a$ and $b$ are available in Refs. [44,45] and Ref. [47] for CLFQM and LCSR(I), respectively. In CCQM and LCSR(II), one needs to apply Eq. (3.15) to get the $q^{2}$ dependence of $F_{0}$. The form-factor

\begin{tabular}{|c|c|c|c|c|c|}
\hline Transition & $\begin{array}{c}\text { CLFQM } \\
{[44,45]}\end{array}$ & $\begin{array}{c}\text { CCQM } \\
{[46]}\end{array}$ & $\begin{array}{c}\operatorname{LCSR}(\mathrm{I}) \\
{[47]}\end{array}$ & $\begin{array}{c}\text { LCSR(II) } \\
{[48]}\end{array}$ & $\begin{array}{c}\operatorname{LCSR}(\mathrm{III}) \\
{[49]}\end{array}$ \\
\hline$D \rightarrow f_{0}(980)$ & $0.51_{-0.05}^{+0.04 a}$ & $0.45 \pm 0.02$ & 0.321 & & \\
\hline$D_{s}^{+} \rightarrow f_{0}(980)$ & $0.52_{-0.01}^{+0.01 \mathrm{~b}}$ & $0.36 \pm 0.02$ & & & \\
\hline$D \rightarrow a_{0}(980)^{\mathrm{c}}$ & & $0.55 \pm 0.02$ & & $0.88 \pm 0.13^{\mathrm{d}}$ & $0.85_{-011}^{+0.10}$ \\
\hline$D \rightarrow a_{0}(1450)$ & $0.51_{-0.02}^{+0.01}$ & & & & $0.94_{-0.03}^{+0.02}$ \\
\hline$D \rightarrow K_{0}^{*}(1430)$ & $0.47_{-0.03}^{+0.02}$ & & & & \\
\hline$D_{s}^{+} \rightarrow K_{0}^{*}(1430)$ & $0.55_{-0.03}^{+0.02}$ & & & & \\
\hline
\end{tabular}
$q^{2}$ dependence in the LCSR(III) calculation is shown in Fig. 3 of Ref. [49].

BESIII has measured the branching fractions of both $D^{0} \rightarrow a_{0}(980)^{-} e^{+} \nu_{e}$ and $D^{+} \rightarrow a_{0}(980)^{0} e^{+} \nu_{e}[50]$. The theoretical calculations depend on the form factors $F_{+}\left(q^{2}\right)$ and $F_{-}\left(q^{2}\right)$ and their $q^{2}$ dependence (see e.g., Ref. [51]). It turns out that the predicted branching fractions for $D \rightarrow a_{0}(980) e^{+} \nu_{e}$ in LCSR(II) [48] are too large by

TABLE III. Form factors $F_{0}^{D S}(0)$ for $D, D_{s} \rightarrow f_{0}(980), a_{0}(980), a_{0}(1450)$ and $K_{0}^{*}(1430)$ transitions in various models.

${ }^{\mathrm{a}}$ For $D \rightarrow f_{0}^{q}$ transition.

${ }^{\mathrm{b}}$ For $D_{s}^{+} \rightarrow f_{0}^{s}$ transition.

${ }^{c}$ It stands for either $D^{0} \rightarrow a_{0}(980)^{-}$or $D^{+} \rightarrow a_{0}(980)^{0}$ transition.

${ }^{\mathrm{d}}$ Use of the relation $F_{+}(0)=F_{+}^{\prime}(0) / 2$ has been made. 
more than a factor of 2 compared to the BESIII experiment (see Table VI of Ref. [49]). Hence, this model is disfavored.

\section{DIAGRAMMATIC AMPLITUDES}

A least model-dependent analysis of heavy meson decays can be carried out in the so-called topological diagram approach. In this diagrammatic scenario, all twobody nonleptonic weak decays of heavy mesons can be expressed in terms of six distinct quark diagrams [52-54]: $T$, the external $W$-emission tree diagram; $C$, the internal $W$-emission; $E$, the $W$-exchange; $A$, the $W$-annihilation; $H$, the horizontal $W$-loop; and $V$, the vertical $W$-loop. The one-gluon exchange approximation of the $H$ graph is the so-called "penguin diagram." These diagrams are classified according to the topologies of weak interactions with all strong interaction effects encoded.

The topological amplitudes for $D \rightarrow S P$ decays have been discussed in [11,14]. Just as $D \rightarrow V P$ decays, one generally has two sets of distinct diagrams for each topology. For example, there are two external $W$-emission and two internal $W$-emission diagrams, depending on whether the emitted particle is an even-party meson or an odd-parity one. Following the convention in $[11,14]$, we shall denote the primed amplitudes $T^{\prime}$ and $C^{\prime}$ for the case when the emitted meson is a scalar one. For the $W$-exchange and $W$ annihilation diagrams with the final state $q_{1} \bar{q}_{2}$, the primed amplitude denotes that the even-parity meson contains the quark $q_{1}$. Since $K_{0}^{*}, a_{0}(1450)$ and the light scalars $\sigma, \kappa, f_{0}(980), a_{0}(980)$ fall into two different $\mathrm{SU}(3)$ flavor nonets, in principle one cannot apply $\mathrm{SU}(3)$ symmetry to relate the topological amplitudes in $D^{+} \rightarrow f_{0}(980) \pi^{+}$to, for example, those in $D^{+} \rightarrow \bar{K}_{0}^{* 0} \pi^{+}$.

In Ref. [14] we have presented the topological amplitude decomposition in $D \rightarrow S P$ decays in two different schemes. In scheme I, light scalar mesons $\sigma, \kappa, a_{0}(980)$ and $f_{0}(980)$ are described by the ground-state $q \bar{q}$ states, while $K_{0}^{*}$ and $a_{0}(1450)$ as excited $q \bar{q}$ states. In scheme II, light scalars are tetraquark states, while $K_{0}^{*}$ and $a_{0}(1450)$ are ground-state $q \bar{q}$. The topological amplitudes for $D \rightarrow S P$ decays are listed in Table IV. The expressions of topological amplitudes are the same in both schemes I and II except for the channels involving $f_{0}$ and $\sigma$. For example,

$$
\begin{gathered}
A\left(D^{+} \rightarrow f_{0} \pi^{+}\right)= \begin{cases}\frac{1}{\sqrt{2}} V_{c d}^{*} V_{u d}\left(T+C^{\prime}+A+A^{\prime}\right) \sin \theta+V_{c s}^{*} V_{u s} C^{\prime} \cos \theta & \text { scheme I, } \\
\frac{1}{\sqrt{2}} V_{c d}^{*} V_{u d}\left(T+C^{\prime}+A+A^{\prime}\right)+\sqrt{2} V_{c s}^{*} V_{u s} C^{\prime} & \text { scheme II, }\end{cases} \\
A\left(D^{+} \rightarrow \sigma \pi^{+}\right)= \begin{cases}\frac{1}{\sqrt{2}} V_{c d}^{*} V_{u d}\left(T+C^{\prime}+A+A^{\prime}\right) \cos \theta-V_{c s}^{*} V_{u s} C^{\prime} \sin \theta & \text { scheme I, } \\
V_{c d}^{*} V_{u d}\left(T+C^{\prime}+A+A^{\prime}\right) & \text { scheme II. }\end{cases}
\end{gathered}
$$

In our numerical estimates, we will take $\theta=30^{\circ}$, saturating the measured upper bound mentioned earlier.

In Table IV the upper part involves only light scalar mesons $\left(f_{0}, a_{0}, \sigma\right.$, and $\left.\kappa\right)$, whereas the lower part involves the $a_{0}(1450)$ and $K_{0}^{*}(1430)$ mesons in the heavier nonet representation. This division is made because the amplitudes of the same topology in these two groups have no a priori relations. In each group we have 15 unknown parameters for the 8 topological amplitudes $T, C, E, A$ and $T^{\prime}, C^{\prime}, E^{\prime}, A^{\prime}$. For neutral scalar mesons $\sigma, f_{0}$ and $a_{0}^{0}$, we cannot set $T^{\prime}=C^{\prime}=0$ even though their vector decay constants vanish. As will be discussed in Sec. VA, $T^{\prime}$ and $C^{\prime}$ do receive nonfactorizable contributions through vertex and spectator-scattering corrections $[55,56]$. Nevertheless, it is naïvely expected that, for example, $\left|T^{\prime}\right| \ll|T|$ and $\left|C^{\prime}\right| \ll|C|$ for charged $a_{0}$. However, as we shall see in Sec. V C, a realistic calculation yields $\left|C^{\prime}\right|>|C|$ instead. At any rate, we have more theory parameters than observables (6 in the upper part and 5 in the lower part of the table), barring a fit.

Since the branching fractions of $f_{0} \rightarrow \pi \pi$ and $\left(f_{0}, a_{0}\right) \rightarrow$ $K \bar{K}$ are unknown, many of the two-body decays in
Table IV cannot be extracted from the data of three-body decays. Nevertheless, the strong couplings such as $g_{f_{0} \rightarrow \pi \pi}, g_{f_{0} \rightarrow K \bar{K}}, g_{a_{0} \rightarrow K \bar{K}}$ and $g_{a_{0} \rightarrow \eta \pi}$ have been inferred from a fit to the data. There are 17 available $D \rightarrow S P \rightarrow P_{1} P_{2} P_{2}$ modes, but there are only 14 data related to $D \rightarrow S P$ and we have 15 parameters to fit. Moreover, since we need to introduce appropriate energy-dependent line shapes for the scalar mesons, it is not conceivable to extract the topological amplitudes from three-body decays as the decay rate cannot be factorized into the topological amplitude squared and the phase space factor. We will come back to this point later.

It is interesting to notice that the current data already imply the importance of $W$-exchange and $W$-annihilation amplitudes. Consider the decays: $D^{0} \rightarrow a_{0}^{+} \pi^{-} \rightarrow K^{+} \bar{K}^{0} \pi^{-}$ and $D^{0} \rightarrow a_{0}^{-} \pi^{+} \rightarrow K^{-} K^{0} \pi^{+}$with the two-body decay amplitudes proportional to $\left(T^{\prime}+E\right)$ and $\left(T+E^{\prime}\right)$, respectively (see Table IV). If the $W$-exchange contributions are negligible, the former mode governed by the amplitude $T^{\prime}$ is expected to have a rate smaller than the latter (cf. Table II). Experimentally, it is the other way around. This is an indication that $E$ and $E^{\prime}$ play some role. 
TABLE IV. Topological amplitudes of various $D \rightarrow S P$ decays. Schemes I has $(\alpha, \beta)=(\sin \theta, \cos \theta)$, and scheme II has $(\alpha, \beta)=(1, \sqrt{2})$ for those modes with one $f_{0}$ and $(0, \sqrt{2})$ for those modes with one $\sigma$. In scheme I, light scalar mesons $\sigma, \kappa, a_{0}(980)$ and $f_{0}(980)$ are described by the $q \bar{q}$ states, while $K_{0}^{*}$ and $a_{0}(1450)$ as excited $q \bar{q}$ states. In scheme II, light scalars are tetraquark states, while $K_{0}^{*}$ and $a_{0}(1450)$ are ground-state $q \bar{q}$. The $f_{0}-\sigma$ mixing angle $\theta$ in the two-quark model is defined in Eq. (3.3). The experimental branching fractions denoted by $\mathcal{B}_{\text {NWA }}$ are taken from Tables I and II. For simplicity, we do not consider the $f_{0}-\sigma$ mixing in the tetraquark model as its value is close to $\pi$ [38].

\begin{tabular}{|c|c|c|}
\hline Decay & Amplitude & $\mathcal{B}_{\mathrm{NWA}}$ \\
\hline$D^{+} \rightarrow f_{0} \pi^{+}$ & $\frac{1}{\sqrt{2}} \alpha V_{c d}^{*} V_{u d}\left(T+C^{\prime}+A+A^{\prime}\right)+\beta V_{c s}^{*} V_{u s} C^{\prime}$ & \\
\hline$\rightarrow f_{0} K^{+}$ & $V_{c d}^{*} V_{u s}\left[\frac{1}{\sqrt{2}} \alpha\left(T+A^{\prime}\right)+\beta A\right]$ & \\
\hline$\rightarrow a_{0}^{+} \bar{K}^{0}$ & $V_{c s}^{*} V_{u d}\left(T^{\prime}+C\right)$ & \\
\hline$\rightarrow a_{0}^{0} \pi^{+}$ & $\frac{1}{\sqrt{2}} V_{c d}^{*} V_{u d}\left(-T-C^{\prime}-A+A^{\prime}\right)$ & \\
\hline$\rightarrow \sigma \pi^{+}$ & $\frac{1}{\sqrt{2}} \beta V_{c d}^{*} V_{u d}\left(T+C^{\prime}+A+A^{\prime}\right)-\alpha V_{c s}^{*} V_{u s} C^{\prime}$ & $(2.1 \pm 0.2) \times 10^{-3}$ \\
\hline$\rightarrow \bar{\kappa}^{0} \pi^{+}$ & $V_{c s}^{*} V_{u d}\left(T+C^{\prime}\right)$ & $\left(3.6_{-2.4}^{+3.0}\right) \%$ \\
\hline$\rightarrow \bar{\kappa}^{0} K^{+}$ & $V_{c s}^{*} V_{u s} T+V_{c d}^{*} V_{u d} A$ & $\left(1.0_{-0.3}^{+0.5}\right) \times 10^{-3}$ \\
\hline$D^{0} \rightarrow f_{0} \pi^{0}$ & $\frac{1}{2} \alpha V_{c d}^{*} V_{u d}\left(-C+C^{\prime}-E-E^{\prime}\right)+\frac{1}{\sqrt{2}} \beta V_{c s}^{*} V_{u s} C^{\prime}$ & \\
\hline$\rightarrow f_{0} \bar{K}^{0}$ & $V_{c s}^{*} V_{u d}\left[\frac{1}{\sqrt{2}} \alpha(C+E)+\beta E^{\prime}\right]$ & \\
\hline$\rightarrow a_{0}^{+} \pi^{-}$ & $V_{c d}^{*} V_{u d}\left(T^{\prime}+E\right)$ & \\
\hline$\rightarrow a_{0}^{-} \pi^{+}$ & $V_{c d}^{*} V_{u d}\left(T+E^{\prime}\right)$ & \\
\hline$\rightarrow a_{0}^{+} K^{-}$ & $V_{c s}^{*} V_{u d}\left(T^{\prime}+E\right)$ & \\
\hline$\rightarrow a_{0}^{0} \bar{K}^{0}$ & $V_{c s}^{*} V_{u d}(C-E) / \sqrt{2}$ & $(2.83 \pm 0.66) \%$ \\
\hline$\rightarrow a_{0}^{-} K^{+}$ & $V_{c d}^{*} V_{u s}\left(T+E^{\prime}\right)$ & \\
\hline$\rightarrow \sigma \pi^{0}$ & $\frac{1}{2} V_{c d}^{*} V_{u d} \beta\left(-C+C^{\prime}-E-E^{\prime}\right)-\frac{1}{\sqrt{2}} \alpha V_{c s}^{*} V_{u s} C^{\prime}$ & $(1.8 \pm 0.3) \times 10^{-4}$ \\
\hline$D_{s}^{+} \rightarrow f_{0} \pi^{+}$ & $\frac{1}{\sqrt{2}} V_{c s}^{*} V_{u d}\left[\sqrt{2} \beta T+\alpha\left(A+A^{\prime}\right)\right]$ & \\
\hline$\rightarrow f_{0} K^{+}$ & $V_{c s}^{*} V_{u s}\left[\beta\left(T+C^{\prime}+A\right)+\frac{1}{\sqrt{2}} \alpha A^{\prime}\right]+\frac{1}{\sqrt{2}} V_{c d}^{*} V_{u d} \alpha C^{\prime}$ & \\
\hline$\rightarrow a_{0}^{0} \pi^{+}$ & $\frac{1}{\sqrt{2}} V_{c s}^{*} V_{u d}\left(-A+A^{\prime}\right)$ & $(0.86 \pm 0.23) \%^{\mathrm{a}}$ \\
\hline$D^{+} \rightarrow a_{0}(1450)^{0} \pi^{+}$ & $\frac{1}{\sqrt{2}} V_{c d}^{*} V_{u d}\left(-T-C^{\prime}-A+A^{\prime}\right)$ & \\
\hline$\rightarrow \bar{K}_{0}^{* 0} \pi^{+}$ & $V_{c s}^{*} V_{u d}\left(T+C^{\prime}\right)$ & $(1.98 \pm 0.22) \%$ \\
\hline$\rightarrow \bar{K}_{0}^{* 0} K^{+}$ & $V_{c s}^{*} V_{u s} T+V_{c d}^{*} V_{u d} A$ & Prohibited \\
\hline$D^{0} \rightarrow a_{0}(1450)^{+} \pi^{-}$ & $V_{c d}^{*} V_{u d}\left(T^{\prime}+E\right)$ & \\
\hline$\rightarrow a_{0}(1450)^{-} \pi^{+}$ & $V_{c d}^{*} V_{u d}\left(T+E^{\prime}\right)$ & \\
\hline$\rightarrow a_{0}(1450)^{-} K^{+}$ & $V_{c d}^{*} V_{u s}\left(T+E^{\prime}\right)$ & \\
\hline$\rightarrow K_{0}^{*-} \pi^{+}$ & $V_{c s}^{*} V_{u d}\left(T+E^{\prime}\right)$ & $(8.8 \pm 1.5) \times 10^{-3}$ \\
\hline$\rightarrow \bar{K}_{0}^{* 0} \pi^{0}$ & $\frac{1}{\sqrt{2}} V_{c s}^{*} V_{u d}\left(C^{\prime}-E^{\prime}\right)$ & $\left(9.5_{-2.8}^{+8.1}\right) \times 10^{-3}$ \\
\hline$\rightarrow K_{0}^{*+} \pi^{-}$ & $V_{c d}^{*} V_{u s}\left(T^{\prime}+E\right)$ & $<4.5 \times 10^{-5}$ \\
\hline$D_{s}^{+} \rightarrow K_{0}^{* 0} \pi^{+}$ & $V_{c d}^{*} V_{u d} T+V_{c s} V_{u s}^{*} A$ & $(8.1 \pm 5.7) \times 10^{-4}$ \\
\hline$\rightarrow \bar{K}_{0}^{* 0} K^{+}$ & $V_{c s}^{*} V_{u d}\left(C^{\prime}+A\right)$ & $(2.8 \pm 0.5) \times 10^{-3}$ \\
\hline
\end{tabular}

${ }^{\text {a }}$ Since the decay amplitudes of $D_{s}^{+} \rightarrow a_{0}^{+} \pi^{0}$ and $D_{s}^{+} \rightarrow a_{0}^{0} \pi^{+}$are the same except an overall negative sign, they have the same rates.

\section{FACTORIZATION APPROACH}

The diagrammatic approach has been applied quite successfully to hadronic decays of charmed mesons into $P P$ and $V P$ final states [57-66]. When generalized to the decay modes involving a scalar meson in the final state, it appears that the current data are still insufficient for us to fully extract the information of all amplitudes. Therefore, we take the naïve factorization formalism as a complementary approach to estimate the rates of these decay modes. In this framework, the $W$-exchange and -annihilation type of contributions will be neglected.

\section{A. Factorizable and nonfactorizable amplitudes}

The factorizable amplitudes for the $D \rightarrow S P$ decays read $X^{(D S, P)}=\left\langle P(q)\left|(V-A)_{\mu}\right| 0\right\rangle\left\langle S(p)\left|(V-A)^{\mu}\right| D\left(p_{D}\right)\right\rangle$, $X^{(D P, S)}=\left\langle S(q)\left|(V-A)_{\mu}\right| 0\right\rangle\left\langle P(p)\left|(V-A)^{\mu}\right| D\left(p_{D}\right)\right\rangle$,

and have the expressions

$$
\begin{aligned}
& X^{(D S, P)}=-f_{P}\left(m_{D}^{2}-m_{S}^{2}\right) F_{0}^{D S}\left(q^{2}\right), \\
& X^{(D P, S)}=f_{S}\left(m_{D}^{2}-m_{P}^{2}\right) F_{0}^{D P}\left(q^{2}\right),
\end{aligned}
$$


TABLE V. Numerical values of the flavor operators $a_{1,2}\left(M_{1} M_{2}\right)$ for $M_{1} M_{2}=S P$ and $P S$ at the scale $\mu=\bar{m}_{c}\left(\bar{m}_{c}\right)=1.3 \mathrm{GeV}$, where use of $c_{1}(\mu)=1.33$ and $c_{2}(\mu)=-0.62$ has been made.

\begin{tabular}{|c|c|c|c|c|c|}
\hline & $f_{0}(500) \pi$ & $\pi f_{0}(500)$ & & $K_{0}^{*}(700) \pi$ & $\pi K_{0}^{*}(700)$ \\
\hline$a_{1}$ & $1.292+0.080 i$ & $0.033-0.056 i$ & $a_{1}$ & $1.292+0.080 i$ & $1.579-0.492 i$ \\
\hline \multirow[t]{2}{*}{$a_{2}$} & $-0.527-0.172 i$ & $-0.070+0.121 i$ & $a_{2}$ & $-0.527-0.172 i$ & $-1.147+0.930 i$ \\
\hline & $f_{0}(980) \pi$ & $\pi f_{0}(980)$ & & $f_{0}(980) K$ & $K f_{0}(980)$ \\
\hline$a_{1}$ & $1.292+0.080 i$ & $0.033-0.056 i$ & $a_{1}$ & $1.295+0.075 i$ & $0.033+0.075 i$ \\
\hline \multirow[t]{2}{*}{$a_{2}$} & $-0.527-0.172 i$ & $-0.070+0.121 i$ & $a_{2}$ & $-0.533-0.162 i$ & $-0.070+0.121 i$ \\
\hline & $a_{0}(980)^{0} \pi$ & $\pi a_{0}(980)^{0}$ & & $a_{0}(980)^{0} K$ & $K a_{0}(980)^{0}$ \\
\hline$a_{1}$ & $1.292+0.080 i$ & $0.037-0.066 i$ & $a_{1}$ & $1.295+0.075 i$ & $0.037-0.066 i$ \\
\hline \multirow[t]{2}{*}{$a_{2}$} & $-0.527-0.172 i$ & $-0.080+0.141 i$ & $a_{2}$ & $-0.533-0.162 i$ & $-0.080+0.141 i$ \\
\hline & $a_{0}(980)^{ \pm} \pi$ & $\pi a_{0}(980)^{ \pm}$ & & $a_{0}(980)^{ \pm} K$ & $K a_{0}(980)^{ \pm}$ \\
\hline$a_{1}$ & $1.292+0.080 i$ & $\pm(-10.04+20.03 i)$ & $a_{1}$ & $1.295+0.075 i$ & $\pm(-10.04+20.03 i)$ \\
\hline \multirow[t]{2}{*}{$a_{2}$} & $-0.527-0.172 i$ & $\pm(23.89-43.14 i)$ & $a_{2}$ & $-0.533-0.162 i$ & $\pm(23.89-43.14 i)$ \\
\hline & $a_{0}(1450) \pi$ & $\pi a_{0}(1450)$ & & $K_{0}^{*}(1430) \pi$ & $\pi K_{0}^{*}(1430)$ \\
\hline$a_{1}$ & $1.292+0.080 i$ & $0.033-0.056 i$ & $a_{1}$ & $1.292+0.080 i$ & $1.692-0.544 i$ \\
\hline$a_{2}$ & $-0.527-0.172 i$ & $-0.071+0.108 i$ & $a_{2}$ & $-0.527-0.172 i$ & $-1.390+1.171 i$ \\
\hline
\end{tabular}

where use of Eqs. (3.7) and (3.12) has been made. Hence,

$$
\begin{aligned}
T & =-a_{1}(S P) f_{P}\left(m_{D}^{2}-m_{S}^{2}\right) F_{0}^{D S}\left(q^{2}\right), \\
C & =-a_{2}(S P) f_{P}\left(m_{D}^{2}-m_{S}^{2}\right) F_{0}^{D S}\left(q^{2}\right), \\
T^{\prime} & =a_{1}(P S) f_{S}\left(m_{D}^{2}-m_{P}^{2}\right) F_{0}^{D P}\left(q^{2}\right), \\
C^{\prime} & =a_{2}(P S) f_{S}\left(m_{D}^{2}-m_{P}^{2}\right) F_{0}^{D P}\left(q^{2}\right) .
\end{aligned}
$$

The primed amplitudes $T^{\prime}$ and $C^{\prime}$ vanish for the neutral scalar mesons such as $\sigma / f_{0}(500), f_{0}(980)$ and $a_{0}(980)^{0}$ as they cannot be produced through the $(V-A)$ current; that is, $f_{S}=0$. Nevertheless, beyond the factorization approximation, contributions proportional to the scalar decay constant $\bar{f}_{S}$ of the scalar meson defined in Eq. (3.7) can be produced from vertex and hard spectator-scattering corrections. It has been shown in Refs. [55,56] that the nonfactorizable amplitudes can be recast to

$$
\begin{aligned}
& T^{\prime}=a_{1}(P S) \bar{f}_{S}\left(m_{D}^{2}-m_{P}^{2}\right) F_{0}^{D P}\left(q^{2}\right), \\
& C^{\prime}=a_{2}(P S) \bar{f}_{S}\left(m_{D}^{2}-m_{P}^{2}\right) F_{0}^{D P}\left(q^{2}\right),
\end{aligned}
$$

for $S=\sigma / f_{0}(500), f_{0}(980)$ and $a_{0}(980)^{0}$, etc., while the expressions of $T^{\prime}$ and $C^{\prime}$ given in Eq. (5.3) are valid for $S=a_{0}^{ \pm}, \kappa / K_{0}^{*}(800)$ and $K_{0}^{*}(1430)$, etc.

\section{B. Flavor operators}

The flavor operators $a_{i}\left(M_{1} M_{2}\right)$ in Eqs. (5.3) and (5.4) are basically the Wilson coefficients in conjunction with short-distance nonfactorizable corrections such as vertex corrections and hard spectator interactions. In general, they have the expressions $[67,68]^{3}$

\footnotetext{
${ }^{3}$ Notice that $a_{1}$ and $a_{2}$ do not receive contributions from penguin contractions.
}

$$
\begin{aligned}
a_{1}\left(M_{1} M_{2}\right)= & \left(c_{1}+\frac{c_{2}}{N_{c}}\right) N_{1}\left(M_{2}\right) \\
& +\frac{c_{2}}{N_{c}} \frac{C_{F} \alpha_{s}}{4 \pi}\left[V_{1}\left(M_{2}\right)+\frac{4 \pi^{2}}{N_{c}} H_{1}\left(M_{1} M_{2}\right)\right], \\
a_{2}\left(M_{1} M_{2}\right)= & \left(c_{2}+\frac{c_{1}}{N_{c}}\right) N_{2}\left(M_{2}\right) \\
& +\frac{c_{1}}{N_{c}} \frac{C_{F} \alpha_{s}}{4 \pi}\left[V_{2}\left(M_{2}\right)+\frac{4 \pi^{2}}{N_{c}} H_{2}\left(M_{1} M_{2}\right)\right],
\end{aligned}
$$

where $c_{i}$ are the Wilson coefficients, $C_{F}=\left(N_{c}^{2}-1\right) /\left(2 N_{c}\right)$ with $N_{c}=3, M_{2}$ is the emitted meson and $M_{1}$ shares the same spectator quark with the $D$ meson. The quantities $V_{i}\left(M_{2}\right)$ account for vertex corrections, $H_{i}\left(M_{1} M_{2}\right)$ for hard spectator interactions with a hard gluon exchange between the emitted meson and the spectator quark of the $D$ meson. The explicit expressions of $V_{1,2}(M)$ and $H_{1,2}\left(M_{1} M_{2}\right)$ in the QCD factorization approach are given in [55]. The expression of the quantities $N_{i}\left(M_{2}\right)$, which are relevant to the factorizable amplitudes, reads

$N_{i}(P)=1, \quad N_{i}(S)= \begin{cases}0, & \text { for } S=\sigma, f_{0}, a_{0}^{0}, \\ 1, & \text { else. }\end{cases}$

Results for the flavor operators $a_{i}\left(M_{1} M_{2}\right)$ with $M_{1} M_{2}=S P$ and $P S$ are shown in Table $\mathrm{V}^{4}$

We see from Eqs. (5.5) and (5.6) that the factorizable contributions to $a_{1}(P S)$ and $a_{2}(P S)$ vanish for $S=\sigma, f_{0}$ and $a_{0}^{0}$. Beyond the factorization approximation, nonfactorizable

\footnotetext{
${ }^{4}$ Studies of $B \rightarrow S P$ decays in QCDF were presented in Refs. $[55,56]$. Here We generalize these works to the $D \rightarrow S P$ decays and obtain the flavor operators given in Table V.
} 
contributions proportional to the decay constant $\bar{f}_{S}$ can be produced from vertex and spectator-scattering corrections $[55,56]$. Therefore, when the strong coupling $\alpha_{s}$ is turned off, the nonfactorizable contributions vanish accordingly. In short, the primed amplitudes $T^{\prime}$ and $C^{\prime}$ are factorizable for $S=a_{0}^{ \pm}, \kappa, K_{0}^{*}$, namely $\left\langle S\left|J^{\mu}\right| 0\right\rangle\left\langle P\left|J_{\mu}^{\prime}\right| D\right\rangle$, whereas they are nonfactorizable for $S=\sigma, f_{0}, a_{0}^{0}$.

Upon an inspection of Table V, we see that (i) the flavor operators $a_{i}(P S)$ and $a_{i}(S P)$ are very different as the former does not receive factorizable contributions [i.e., $N_{i}(S)=0$ ], and (ii) while $a_{1}(S P)$ and $a_{2}(S P)$ are similar for any light and heavy scalar mesons, namely $a_{1}(S P) \approx 1.29 \pm 0.08 i$ and $a_{2}(S P) \approx-0.53-0.17 i, a_{1}(P S)$ and $a_{2}(P S)$ vary from neutral to the charged ones as shown in Table VI. One may wonder why the flavor operators $a_{1,2}\left(\pi a_{0}^{ \pm}\right)$are much greater than $a_{1,2}\left(\pi a_{0}^{0}\right)$. As noticed in Eqs. (5.3) and (5.4), the nonfactorizable amplitudes are proportional to $a_{1,2}\left(\pi a_{0}^{ \pm}\right) f_{a_{0}^{ \pm}}$ for charged $a_{0}^{ \pm}$and to $a_{1,2}\left(\pi a_{0}^{0}\right) \bar{f}_{a_{0}}$ for neutral $a_{0}^{0}$. Hence, $a_{1,2}\left(\pi a_{0}^{ \pm}\right) / a_{1,2}\left(\pi a_{0}^{0}\right)=\bar{f}_{a_{0}} / f_{a_{0}^{ \pm}} \gg 1$. We see from Table VI that $a_{1,2}(P S)$ become larger when the decay constants become smaller.

\section{Implications}

Naïvely it is expected that $\left|T^{\prime}\left(\pi^{-} a_{0}^{+}\right)\right| \ll\left|T\left(a_{0}^{-} \pi^{+}\right)\right|$ because $f_{\pi} \gg f_{a_{0}^{+}}$and $\left|C^{\prime}\left(\pi^{+} \bar{\kappa}^{0}\right)\right|<\left|C\left(\pi^{+} f_{0}\right)\right|$ due to the fact that $f_{\pi}>f_{\kappa}$. Although we are not able to extract the topological amplitudes of $D \rightarrow S P$ from the experimental data of three-body $D \rightarrow P_{1} P_{2} P_{3}$ decays, we can use the theoretical calculations to see their sizes and relative phases. From Eq. (5.3) we have

$$
\begin{aligned}
T\left(f_{0} \pi^{+}\right) & =-a_{1}\left(f_{0} \pi\right) f_{\pi}\left(m_{D}^{2}-m_{f_{0}}^{2}\right) F_{0}^{D f_{0}}\left(m_{\pi}^{2}\right), \\
C\left(f_{0} \pi^{0}\right) & =-a_{2}\left(f_{0} \pi\right) f_{\pi}\left(m_{D}^{2}-m_{f_{0}}^{2}\right) F_{0}^{D f_{0}}\left(m_{\pi}^{2}\right), \\
T^{\prime}\left(\pi^{-} a_{0}^{+}\right) & =a_{1}\left(\pi a_{0}^{+}\right) f_{a_{0}^{+}}\left(m_{D}^{2}-m_{\pi}^{2}\right) F_{0}^{D \pi}\left(m_{a_{0}}^{2}\right), \\
C^{\prime}\left(\pi^{0} f_{0}^{0}\right) & =a_{2}\left(\pi f_{0}\right) \bar{f}_{f_{0}}\left(m_{D}^{2}-m_{\pi}^{2}\right) F_{0}^{D \pi}\left(m_{f_{0}}^{2}\right), \\
C^{\prime}\left(\pi^{+} \bar{\kappa}^{0}\right) & =a_{2}(\pi \kappa) f_{\kappa}\left(m_{D}^{2}-m_{\pi}^{2}\right) F_{0}^{D \pi}\left(m_{\kappa}^{2}\right) .
\end{aligned}
$$

Using the flavor operators given in Table $\mathrm{V}$, form factors $F^{D S}$ listed in Table III and $F^{D P}\left(q^{2}\right)$ evaluated in the

TABLE VI. Same as Table V except for the flavor operators $a_{1,2}(P S)$ with $P=\pi$. For neutral scalar mesons $\sigma, f_{0}, a_{0}^{0}$, the vector decay constant $f_{S}$ is replaced by the scalar decay constant $\bar{f}_{S}$.

\begin{tabular}{lccc}
\hline \hline$S$ & $f_{S}(\mathrm{MeV})$ & $a_{1}(P S)$ & $a_{2}(P S)$ \\
\hline$\sigma, f_{0}, a_{0}^{0}$ & $350-370$ & $\sim 0.035-0.060 i$ & $\sim-0.075+0.130 i$ \\
$\bar{\kappa}$ & 45.5 & $1.58-0.49 i$ & $-1.15+0.93 i$ \\
$\bar{K}_{0}^{*}$ & 35.3 & $1.69-0.54 i$ & $-1.39+1.17 i$ \\
$a_{0}^{-}$ & 1.3 & $10-20 i$ & $-24+43 i$ \\
\hline \hline
\end{tabular}

covariant confining quark model [69], we find numerically (in units of $10^{-6} \mathrm{GeV}$ ),

$$
\begin{aligned}
T\left(f_{0} \pi^{+}\right) & =1.80 e^{-i 186^{\circ}}, \quad C\left(f_{0} \pi^{0}\right)=0.77 e^{-i 18^{\circ},} \\
T^{\prime}\left(\pi^{-} a_{0}^{+}\right) & =0.55 e^{i 117^{\circ}}, \\
C^{\prime}\left(\pi^{0} f_{0}\right) & =0.99 e^{i 120^{\circ}}, \quad C^{\prime}\left(\pi^{+} \bar{\kappa}^{0}\right)=1.26 e^{i 141^{\circ} .}
\end{aligned}
$$

For heavier scalar mesons we find

$$
\begin{aligned}
T\left(K_{0}^{*-} \pi^{+}\right) & =0.70 e^{-i 177^{\circ}}, \\
T^{\prime}\left(\pi^{-} K_{0}^{*+}\right) & =1.29 e^{-i 18^{\circ}} \\
C^{\prime}\left(\pi^{0} \bar{K}_{0}^{* 0}\right) & =1.32 e^{i 140^{\circ}} \\
T\left(a_{0}(1450)^{0} \pi^{+}\right) & =0.93 e^{-i 177^{\circ}} \\
T^{\prime}\left(\pi^{-} a_{0}(1450)^{+}\right) & =0.59 e^{i 121^{\circ}} \\
C^{\prime}\left(\pi^{0} a_{0}(1450)^{0}\right) & =1.21 e^{i 123^{\circ}}
\end{aligned}
$$

In the light scalar meson sector, we have $|T|>\left|T^{\prime}\right|$ and $|C|<\left|C^{\prime}\right|$ rather than $|T| \gg\left|T^{\prime}\right|$ and $|C|>\left|C^{\prime}\right|$. For scalar mesons in the higher nonet representation, we find $\left|T^{\prime}\right|>$ $\left|C^{\prime}\right|>|T|$ with $|T|$ being suppressed as the mass term $\left(m_{D}^{2}-m_{S}^{2}\right)$ becomes smaller when $S$ becomes heavier.

\section{Flatté line shape}

To describe three-body decays we need to introduce a line shape of the scalar resonance. Normally we use the relativistic Breit-Wigner line shape to describe the scalar resonance contributions to three-body decays $D \rightarrow S P \rightarrow$ $P_{1} P_{2} P$ :

$$
T^{\mathrm{BW}}(s)=\frac{1}{s-m_{R}^{2}+i m_{R} \Gamma_{R}(s)},
$$

with

$$
\Gamma_{R}(s)=\Gamma_{R}^{0}\left(\frac{q}{q_{0}}\right) \frac{m_{R}}{\sqrt{s}}
$$

where $q=\left|\vec{p}_{1}\right|=\left|\vec{p}_{2}\right|$ is the c.m. momentum in the rest frame of $R, q_{0}$ the value of $q$ when $s$ is equal to $m_{R}^{2}$. However, this parametrization is not suitable to describe the decay of $f_{0}(980)$ or $a_{0}(980)$ into $K \bar{K}$ as $m\left(K^{+}\right)+m\left(K^{-}\right)=$ 987.4 $\mathrm{MeV}$ and $m\left(K^{0}\right)+m\left(\bar{K}^{0}\right)=995.2 \mathrm{MeV}$ are near threshold. In other words, one has to take the threshold effect into account. Since $f_{0}(980)$ couples strongly to the channel $K \bar{K}$ as well as to the channel $\pi \pi$, they can be described by a coupled channel formula, the so-called Flatté line shape [70]

$$
T_{f_{0}}^{\text {Flatte }}(s)=\frac{1}{s-m_{f_{0}}^{2}+i\left[g_{f_{0} \rightarrow \pi \pi}^{2} \rho_{\pi \pi}(s)+g_{f_{0} \rightarrow K \bar{K}}^{2} \rho_{K \bar{K}}(s)\right]},
$$


with the phase space factor

$\rho_{a b}=\frac{1}{16 \pi}\left(1-\frac{\left(m_{a}+m_{b}\right)^{2}}{s}\right)^{1 / 2}\left(1-\frac{\left(m_{a}-m_{b}\right)^{2}}{s}\right)^{1 / 2}$,

so that

$$
\begin{aligned}
\rho_{K \bar{K}}(s) & =\rho_{K^{+} K^{-}}(s)+\rho_{K^{0} \bar{K}^{0}}(s) \\
& =\frac{1}{16 \pi}\left(\sqrt{1-\left(4 m_{K^{ \pm}}^{2} / s\right)}+\sqrt{1-\left(4 m_{K^{0}}^{2} / s\right)}\right), \\
\rho_{\pi \pi}(s) & =\rho_{\pi^{+} \pi^{-}}(s)+\frac{1}{2} \rho_{\pi^{0} \pi^{0}}(s) \\
& =\frac{1}{16 \pi}\left(\sqrt{1-\left(4 m_{\pi^{ \pm}}^{2} / s\right)}+\frac{1}{2} \sqrt{1-\left(4 m_{\pi^{0}}^{2} / s\right)}\right),
\end{aligned}
$$

and $\rho \rightarrow i \sqrt{-\rho^{2}}$ when below the threshold, i.e., $s<4 m_{K}^{2}$ for $\rho_{K \bar{K}}$. The dimensionful coupling constants in Eq. (5.12) are

$$
\begin{aligned}
g_{f_{0} \rightarrow \pi \pi} & \equiv g_{f_{0} \rightarrow \pi^{+} \pi^{-}}=\sqrt{2} g_{f_{0} \rightarrow \pi^{0} \pi^{0}}, \\
g_{f_{0} \rightarrow K \bar{K}} & \equiv g_{f_{0} \rightarrow K^{+} K^{-}}=g_{f_{0} \rightarrow K^{0} \bar{K}^{0}} .
\end{aligned}
$$

Likewise, $a_{0}(980)$ couples strongly to $K \bar{K}$ and $\eta \pi$

$$
T_{a_{0}}^{\text {Flatte }}(s)=\frac{1}{s-m_{a_{0}}^{2}+i\left[g_{a_{0} \rightarrow \eta \pi}^{2} \rho_{\eta \pi}(s)+g_{a_{0} \rightarrow K \bar{K}}^{2} \rho_{K \bar{K}}(s)\right]} .
$$

with

$$
\rho_{\eta \pi}(s)=\frac{1}{16 \pi}\left(1-\frac{\left(m_{\eta}-m_{\pi}\right)^{2}}{s}\right)^{1 / 2}\left(1-\frac{\left(m_{\eta}+m_{\pi}\right)^{2}}{s}\right)^{1 / 2} .
$$

It is important to check whether $g_{f_{0} \rightarrow \pi \pi}$ and $g_{f_{0}, a_{0} \rightarrow K \bar{K}}$ can be interpreted as the strong couplings of $f_{0}$ to $\pi \pi$ and $K \bar{K}$, respectively. Using the formula

$$
\Gamma\left(f_{0} \rightarrow \pi^{+} \pi^{-}\right)=\frac{p_{c}}{8 \pi m_{f_{0}}^{2}} g_{f_{0} \rightarrow \pi^{+} \pi^{-}}^{2},
$$

with $p_{c}$ being the c.m. momentum of the pion in the rest frame of $f_{0}$, it is easily seen that the term $g_{f_{0} \rightarrow \pi \pi}^{2} \rho_{\pi \pi}\left(m_{f_{0}}^{2}\right)$ in Eq. (5.12) is identical to $m_{f_{0}}\left(\Gamma\left(f_{0} \rightarrow \pi^{+} \pi^{-}\right)+\right.$ $\left.\Gamma\left(f_{0} \rightarrow \pi^{0} \pi^{0}\right)\right)$. Therefore, we are sure that $g_{f_{0} \rightarrow \pi \pi}$ is the strong coupling appearing in the matrix element $\left\langle\pi^{+} \pi^{-} \mid f_{0}\right\rangle$. The strong couplings $g_{f_{0}, a_{0} \rightarrow K \bar{K}}, g_{f_{0} \rightarrow \pi \pi}$ and $g_{a_{0} \rightarrow \eta \pi}$ have been extracted from fits to the experimental data. In this work we shall use

$$
\begin{aligned}
g_{f_{0} \rightarrow K \bar{K}} & =(3.54 \pm 0.05) \mathrm{GeV}, \\
g_{a_{0} \rightarrow K \bar{K}} & =(3.77 \pm 0.42) \mathrm{GeV}, \\
g_{f_{0} \rightarrow \pi \pi} & =(1.5 \pm 0.1) \mathrm{GeV}, \\
g_{a_{0} \rightarrow \eta \pi} & =(2.54 \pm 0.16) \mathrm{GeV},
\end{aligned}
$$

where the values of $g_{f_{0} \rightarrow K \bar{K}}$ and $g_{f_{0} \rightarrow \pi \pi}$ are taken from Ref. [6], dominated by the Dalitz plot analysis of $e^{+} e^{-} \rightarrow$ $\pi^{0} \pi^{0} \gamma$ performed by KLOE [71]. The couplings $g_{a_{0} \rightarrow K \bar{K}}$ and $g_{a_{0} \rightarrow \pi \eta}$ are taken from the analysis of the decay $D^{0} \rightarrow$ $K_{S}^{0} K^{+} K^{-}$by BESIII [6]. ${ }^{5}$ Note the result for the coupling $g_{f_{0} \rightarrow \pi \pi}$ is consistent with the value of $1.33_{-0.26}^{+0.29} \mathrm{GeV}$ extracted from Belle's measurement of the partial width of $f_{0}(980) \rightarrow \pi^{+} \pi^{-}$[73].

The partial widths can be inferred from the strong couplings listed in Eq. (5.19) as

$$
\begin{aligned}
& \Gamma\left(f_{0}(980) \rightarrow \pi \pi\right)=(65.7 \pm 8.8) \mathrm{MeV}, \\
& \Gamma\left(a_{0}(980) \rightarrow \eta \pi\right)=(85.2 \pm 10.7) \mathrm{MeV},
\end{aligned}
$$

though they are not directly measured.

\section{E. Line shape for $\sigma / f_{\mathbf{0}}(\mathbf{5 0 0})$}

As stressed in Ref. [74], the scalar resonance $\sigma / f_{0}(500)$ is very broad and cannot be described by the usual BreitWigner line shape. Its partial wave amplitude does not resemble a Breit-Wigner shape with a clear peak and a simultaneous steep rise in the phase. The mass and width of the $\sigma$ resonance are identified from the associated pole position $\sqrt{s_{\sigma}}$ of the partial wave amplitude in the second Riemann sheet as $\sqrt{s_{\sigma}}=m_{\sigma}-i \Gamma_{\sigma} / 2$ [74]. We shall follow the LHCb Collaboration [75] to use a simple pole description

$T_{\sigma}(s)=\frac{1}{s-s_{\sigma}}=\frac{1}{s-m_{\sigma}^{2}+\Gamma_{\sigma}^{2}(s) / 4+i m_{\sigma} \Gamma_{\sigma}(s)}$,

with $\sqrt{s_{\sigma}}=m_{\sigma}-i \Gamma_{\sigma} / 2$ and

$$
\Gamma_{\sigma}(s)=\Gamma_{\sigma}^{0}\left(\frac{q}{q_{0}}\right) \frac{m_{\sigma}}{\sqrt{s}} .
$$

Using the isobar description of the $\pi^{+} \pi^{-} S$-wave to fit the $B^{+} \rightarrow \pi^{+} \pi^{-} \pi^{+}$decay data, the LHCb Collaboration found [75]

\footnotetext{
${ }^{5}$ From the amplitude analysis of the $\chi_{c 1} \rightarrow \eta \pi^{+} \pi^{-}$decay, BESIII obtained another set of couplings: $g_{a_{0} \rightarrow \eta \pi}=(4.14 \pm 0.02) \mathrm{GeV}$ and $g_{a_{0} \rightarrow K \bar{K}}=(3.91 \pm 0.02) \mathrm{GeV}$ [72]. However, this set of couplings is not appealing for two reasons: (a) the large coupling constant $g_{a_{0} \rightarrow \eta \pi}$ will yield too large partial width $\Gamma_{\eta \pi}=222 \mathrm{MeV}$, recalling that the total width of $a_{0}(980)$ lies in the range of 50 to $100 \mathrm{MeV}$ [1], and (b) it is commonly believed that $a_{0}(980)$ couples more strongly to $K \bar{K}$ than to $\eta \pi$, especially in the scenario in which $a_{0}(980)$ is a $K \bar{K}$ molecular state.
} 


$$
\sqrt{s_{\sigma}}=(563 \pm 10)-i(350 \pm 13) \mathrm{MeV},
$$

consistent with the PDG value of $\sqrt{s_{\sigma}}=(400-550)-$ $i(200-350) \mathrm{MeV}$ [1].

In principle, we could also use a similar pole shape $T_{\kappa}(s)$

$T_{\kappa}(s)=\frac{1}{s-s_{\kappa}}=\frac{1}{s-m_{\kappa}^{2}+\Gamma_{\kappa}^{2}(s) / 4+i m_{\kappa} \Gamma_{\kappa}(s)}$.

to describe the broad resonance $\kappa / K_{0}^{*}(700)$ and follow [76] to use the latest result

$$
\sqrt{s_{\kappa}}=(648 \pm 7)-i(280 \pm 16) \mathrm{MeV},
$$

determined from a dispersive data analysis. However, we find that this line shape together with the above pole mass and width will yield a very huge and unreasonable result for the finite-width correction to $D^{+} \rightarrow \bar{\kappa}^{0} \pi^{+}$(see Sec. VIB below). Hence, we will use the usual Breit-Wigner line shape for $\kappa / K_{0}^{*}(700)$ and take the Breit-Wigner mass and width [1]

$$
\begin{aligned}
& m_{K_{0}^{*}(700)}^{\mathrm{BW}}=845 \pm 17 \mathrm{MeV}, \\
& \Gamma_{K_{0}^{*}(700)}^{\mathrm{BW}}=468 \pm 30 \mathrm{MeV} .
\end{aligned}
$$

\section{F. Three-body decays}

We take $D^{+} \rightarrow \sigma \pi^{+} \rightarrow \pi^{+} \pi^{-} \pi^{+}$as an example to illustrate the calculation for the three-body rate. The two-body decay amplitude for $D^{+} \rightarrow \sigma\left(m_{12}\right) \pi^{+}$with $m_{12}$ $\left(m_{12}^{2} \equiv\left(p_{1}+p_{2}\right)^{2}\right)$ being the invariant mass of the $\sigma$ is given by

$$
\begin{aligned}
& A\left(D^{+} \rightarrow \sigma\left(m_{12}\right) \pi^{+}\right) \\
& \quad=\frac{G_{F}}{\sqrt{2}} V_{c d}^{*} V_{u d}\left[-a_{1}(\sigma \pi) f_{\pi}\left(m_{D}^{2}-s\right) F_{0}^{D \sigma}\left(m_{\pi}^{2}\right)\right. \\
& \left.\quad+a_{2}(\pi \sigma) \bar{f}_{\sigma}\left(m_{D}^{2}-m_{\pi}^{2}\right) F_{0}^{D \pi}(s)\right] .
\end{aligned}
$$

Denoting $\mathcal{A}_{\sigma} \equiv A\left(D^{+} \rightarrow \sigma \pi^{+} \rightarrow \pi^{+}\left(p_{1}\right) \pi^{-}\left(p_{2}\right) \pi^{+}\left(p_{3}\right)\right)$, we have

$$
\begin{aligned}
\mathcal{A}_{\sigma}= & g^{\sigma \rightarrow \pi^{+} \pi^{-}} F\left(s_{12}, m_{\sigma}\right) T_{\sigma}\left(s_{12}\right) A\left(D^{+} \rightarrow \sigma\left(s_{12}\right) \pi^{+}\right) \\
& +\left(s_{12} \leftrightarrow s_{23}\right),
\end{aligned}
$$

where the $\sigma$ line shape $T_{\sigma}$ is given by Eq. (5.21). When $\sigma$ is off the mass shell, especially when $s_{12}$ is approaching the upper bound of $\left(m_{D}-m_{\pi}\right)^{2}$, it is necessary to account for the off-shell effect. For this purpose, we shall follow [77] to introduce a form factor $F\left(s, m_{R}\right)$ parametrized as

$$
F\left(s, m_{R}\right)=\left(\frac{\Lambda^{2}+m_{R}^{2}}{\Lambda^{2}+s}\right)^{n},
$$

with the cutoff $\Lambda$ not far from the resonance,

$$
\Lambda=m_{R}+\beta \Lambda_{\mathrm{QCD}}
$$

where the parameter $\beta$ is expected to be of order unity. We shall use $n=1, \Lambda_{\mathrm{QCD}}=250 \mathrm{MeV}$ and $\beta=1.0 \pm 0.2$ in subsequent calculations.

The decay rate then reads

$$
\begin{aligned}
\Gamma\left(D^{+} \rightarrow \sigma \pi^{+} \rightarrow \pi^{+} \pi^{-} \pi^{+}\right)= & \frac{1}{2} \frac{1}{(2 \pi)^{3} 32 m_{D}^{3}} \int d s_{12} d s_{23}\left\{\frac{\left|g^{\sigma \rightarrow \pi^{+} \pi^{-}}\right|^{2} F\left(s_{12}, m_{\sigma}\right)^{2}}{\left(s_{12}-m_{\sigma}^{2}+\Gamma_{\sigma}\left(s_{12}\right) / 4\right)^{2}+m_{\sigma}^{2} \Gamma_{\sigma}^{2}\left(s_{12}\right)}\left|A\left(D^{+} \rightarrow \sigma\left(m_{12}\right) \pi^{+}\right)\right|^{2}\right. \\
& \left.+\left(s_{12} \leftrightarrow s_{23}\right)+\text { interference }\right\}
\end{aligned}
$$

where the factor of $\frac{1}{2}$ accounts for the identical particle effect. The coupling constant $g^{\sigma \rightarrow \pi^{+} \pi^{-}}$is determined by the relation

$$
\Gamma_{\sigma \rightarrow \pi^{+} \pi^{-}}=\frac{p_{c}}{8 \pi m_{\sigma}^{2}} g_{\sigma \rightarrow \pi^{+} \pi^{-}}^{2}
$$

\section{RESULTS AND DISCUSSION}

In Tables VII and VIII we have calculated two-body $D \rightarrow S P$ and three-body $D \rightarrow S P \rightarrow P_{1} P_{2} P$ decays, respectively, in schemes I and II using the factorization approach with $W$-exchange and $W$-annihilation being neglected. We see from Table IV that the decay modes $D^{+} \rightarrow a_{0}^{+} \bar{K}^{0}, \bar{\kappa} \pi^{+}$and $\bar{K}_{0}^{*} \pi^{+}$are free of $W$-annihilation contributions and they are ideal for testing the validity of the factorization approach. From Table VIII it is evident that the calculated rates of $D^{+} \rightarrow \bar{\kappa} \pi^{+} \rightarrow K_{S} \pi^{0} \pi^{+}$and $D^{+} \rightarrow \bar{K}_{0}^{* 0} \pi^{+} \rightarrow(K \pi)^{0} \pi^{+}$in scheme II are in agreement with experiment. These modes are governed by the topologies $T+C^{\prime}$ which interfere constructively. This is in contrast to the Cabibbo-favored (CF) $D^{+} \rightarrow \bar{K}^{0} \pi^{+}$decay in the $P P$ sector where $T$ and $C$ contribute destructively. For $\left(D^{+}, D_{0}, D_{s}^{+}\right) \rightarrow f_{0} P ; f_{0} \rightarrow P_{1} P_{2}$, predictions in scheme II are improved over that in scheme I and the discrepancies presumably arise from the $W$-exchange or 
TABLE VII. Branching fractions for various $D \rightarrow S P$ decays calculated in schemes I and II. The upper part involves only light scalar mesons $\left(f_{0}, a_{0}, \sigma\right.$, and $\left.\kappa\right)$, whereas the lower part involves the $a_{0}(1450)$ and $K_{0}^{*}(1430)$ mesons in the heavier nonet representation. The theoretical calculations are done in the factorization approach with both $W$-exchange and $W$-annihilation amplitudes being neglected. In scheme I, $K_{0}^{*}$ and $a_{0}(1450)$ are excited $q \bar{q}$ states. Hence, their predictions are not presented here. The $f_{0}-\sigma$ mixing angle $\theta$ is taken to be $30^{\circ}$ for scheme $\mathrm{I}$.

\begin{tabular}{|c|c|c|c|}
\hline Decay & Scheme I & Scheme II & $\mathcal{B}_{\mathrm{NWA}}$ \\
\hline$D^{+} \rightarrow \sigma \pi^{+}$ & $2.6 \times 10^{-3}$ & $4.6 \times 10^{-3}$ & $(2.1 \pm 0.2) \times 10^{-3}$ \\
\hline$\rightarrow \bar{\kappa}^{0} \pi^{+}$ & $6.1 \%$ & $6.1 \%$ & $\left(3.6_{-2.4}^{+3.0}\right) \%$ \\
\hline$\rightarrow \bar{\kappa}^{0} K^{+}$ & $1.1 \times 10^{-3}$ & $1.1 \times 10^{-3}$ & $\left(1.0_{-0.3}^{+0.5}\right) \times 10^{-3}$ \\
\hline$D^{0} \rightarrow a_{0}^{0} \bar{K}^{0}$ & $4.2 \times 10^{-3}$ & $4.2 \times 10^{-3}$ & $(2.83 \pm 0.66) \%$ \\
\hline$\rightarrow \sigma \pi^{0}$ & $3.2 \times 10^{-5}$ & $7.8 \times 10^{-5}$ & $(1.8 \pm 0.3) \times 10^{-4}$ \\
\hline$D_{s}^{+} \rightarrow a_{0}^{0} \pi^{+}$ & 0 & 0 & $(0.86 \pm 0.23) \%$ \\
\hline$D^{+} \rightarrow \bar{K}_{0}^{* 0} \pi^{+}$ & & $2.19 \%$ & $(1.98 \pm 0.22) \%$ \\
\hline$D^{0} \rightarrow K_{0}^{*-} \pi^{+}$ & & $2.1 \times 10^{-3}$ & $(8.8 \pm 1.5) \times 10^{-3}$ \\
\hline$\rightarrow \bar{K}_{0}^{* 0} \pi^{0}$ & & $2.1 \times 10^{-3}$ & $\left(9.5_{-28}^{+8.1}\right) \times 10^{-3}$ \\
\hline$\rightarrow K_{0}^{*+} \pi^{-}$ & & $1.1 \times 10^{-5}$ & $<4.5 \times 10^{-5}$ \\
\hline$D_{s}^{+} \rightarrow K_{0}^{* 0} \pi^{+}$ & & $2.9 \times 10^{-4}$ & $(8.1 \pm 5.7) \times 10^{-4}$ \\
\hline$\rightarrow \bar{K}_{0}^{* 0} K^{+}$ & & $3.1 \times 10^{-3}$ & $(2.8 \pm 0.5) \times 10^{-3}$ \\
\hline
\end{tabular}

$W$-annihilation amplitude. This implies that the tetraquark picture for light scalars works better than the quarkantiquark scenario.

Upon an inspection of Table VII, the reader may wonder (i) why the branching fractions for $D \rightarrow\left(f_{0}, \sigma\right) P$ decays in scheme II are always larger than that in scheme I except for $D^{0} \rightarrow f_{0} \pi^{0}$, and (ii) why the predicted branching fractions of $D^{+} \rightarrow \sigma \pi^{+}$and $D^{+} \rightarrow \bar{\kappa}^{0} \pi^{+}$are larger than experimental data, while the corresponding three-body decays agree with the measurements. For (i), we see from Table IV and also Eq. (4.1) that the $W$-emission decay amplitude involving $\sigma$ is suppressed by a factor of $\cos \theta / \sqrt{2}$ in scheme I relative to that in scheme II, while it is suppressed by a factor of $\sin \theta$ for the $W$-emission decay amplitude involving $f_{0}(980)$. As a consequence of our choice of $\theta=30^{\circ}$, the branching fractions for $D \rightarrow\left(f_{0}, \sigma\right) P$ in scheme II are always larger than scheme I except for $D^{0} \rightarrow f_{0} \pi^{0}$. For (ii), it has something to do with the finite-width effects of $\sigma$ and $\kappa$ as they are both very broad. We shall see in Sec. VI B that the extraction of $\mathcal{B}(D \rightarrow S P)$ from the data is affected by the broad widths of both $\sigma$ and $\kappa$.

\section{A. W-annihilation amplitude}

In the factorization calculations presented in Tables VII and VIII, we have neglected both $W$-exchange and $W$-annihilation amplitudes. The $D_{s}^{+} \rightarrow a_{0}^{+} \pi^{0}+a_{0}^{0} \pi^{+}$mode recently observed by BESIII [5] proceeds only through the $W$-annihilation amplitudes. However, its branching fraction at a percent level is much larger than the other two $W$-annihilation channels $D_{s}^{+} \rightarrow \omega \pi^{+}$and $\rho^{0} \pi^{+}$ whose branching fractions are $(1.92 \pm 0.30) \times 10^{-3}$ and $(1.9 \pm 1.2) \times 10^{-4}$, respectively [1]. This implies that $|A(S P)|>|A(V P)|$. In other words, the $W$-annihilation amplitude plays a more significant role in the $S P$ sector than in the $V P$ one.

Consider the decay amplitude of $D_{s}^{+} \rightarrow a_{0}^{0} \pi^{+}$and the $W$-annihilation contribution to $D_{s}^{+} \rightarrow f_{0} \pi^{+}$(in scheme II)

$$
\begin{aligned}
\mathcal{A}\left(D_{s}^{+} \rightarrow a_{0}^{0} \pi^{+}\right) & =\frac{1}{\sqrt{2}} V_{c s}^{*} V_{u d}\left(-A+A^{\prime}\right), \\
\mathcal{A}\left(D_{s}^{+} \rightarrow f_{0} \pi^{+}\right)_{\mathrm{ann}} & =\frac{1}{\sqrt{2}} V_{c s}^{*} V_{u d}\left(A+A^{\prime}\right) .
\end{aligned}
$$

Following the $G$-parity argument given in Ref. [57], it is obvious that the direct $W$-annihilation process through $c \bar{s} \rightarrow W \rightarrow u \bar{d}$ is allowed in $D_{s}^{+} \rightarrow f_{0} \pi^{+}$decay but not in $D_{s}^{+} \rightarrow a_{0}^{0} \pi^{+}$decay as $G(u \bar{d})=-, G\left(a_{0} \pi\right)=+$ and $G\left(f_{0} \pi\right)=-$. This means that short-distance $W$-annihilation contributions respect the relation $A^{\prime}=A$, contrary to the naïve expectation. Hence, one needs large long-distance $W$-annihilation which yields $A^{\prime}=-A$. Since $D_{s}^{+} \rightarrow \rho^{+} \eta$ has the largest branching fraction of $(8.9 \pm 0.8) \%$ among the CF $D_{s}^{+} \rightarrow V P$ decays [1], it is conceivable that longdistance contribution from the weak decays $D_{s}^{+} \rightarrow \rho^{+} \eta$ followed by the resonantlike final-state rescattering of $\rho^{+} \eta \rightarrow a_{0}^{0} \pi^{+}$(see Fig. 1), which has the same topology as $W$-annihilation, may explain the large $W$-annihilation rate. ${ }^{6}$ It is customary to evaluate the final-state rescattering contribution, Fig. 1, at the hadron level manifested in Fig. 2. One of the diagrams, namely, the triangle graph in Fig. 2(b) has been evaluated recently in [78,79]. It yields a major contribution to $D_{s}^{+} \rightarrow a_{0}^{0} \pi^{+}$owing to the large

\footnotetext{
${ }^{6}$ The hadronic weak decays $D_{s}^{+} \rightarrow \rho^{+} \eta^{\prime}, \bar{K}^{* 0} K^{+}$and $\bar{K}^{0} K^{*+}$ followed by final-state rescattering will also contribute to $D_{s}^{+} \rightarrow a_{0}^{0} \pi^{+}$.
} 
TABLE VIII. Branching fractions of various $D \rightarrow S P \rightarrow P_{1} P_{2} P$ decays calculated in schemes I and II. For simplicity and convenience, we have dropped the mass identification for $f_{0}(980), a_{0}(980)$ and $K_{0}^{*}(1430)$. Data are taken from Tables I and II. In scheme I, $K_{0}^{*}$ and $a_{0}(1450)$ are excited $q \bar{q}$ states. Hence, their predictions are not presented here. The $f_{0}-\sigma$ mixing angle $\theta$ is taken to be $30^{\circ}$ for scheme I.

\begin{tabular}{|c|c|c|c|}
\hline$D \rightarrow S P ; S \rightarrow P_{1} P_{2}$ & Scheme I & Scheme II & Experiment \\
\hline$D^{+} \rightarrow f_{0} \pi^{+} ; f_{0} \rightarrow \pi^{+} \pi^{-}$ & $7.6 \times 10^{-5}$ & $2.2 \times 10^{-4}$ & $(1.56 \pm 0.33) \times 10^{-4}$ \\
\hline$D^{+} \rightarrow f_{0} K^{+} ; f_{0} \rightarrow \pi^{+} \pi^{-}$ & $3.6 \times 10^{-7}$ & $1.2 \times 10^{-5}$ & $(4.4 \pm 2.6) \times 10^{-5}$ \\
\hline$D^{+} \rightarrow f_{0} K^{+} ; f_{0} \rightarrow K^{+} K^{-}$ & $2.5 \times 10^{-7}$ & $8.4 \times 10^{-6}$ & $(1.23 \pm 0.02) \times 10^{-5}$ \\
\hline$D^{+} \rightarrow \sigma \pi^{+} ; \sigma \rightarrow \pi^{+} \pi^{-}$ & $4.9 \times 10^{-4}$ & $1.7 \times 10^{-3}$ & $(1.38 \pm 0.12) \times 10^{-3}$ \\
\hline$D^{+} \rightarrow \bar{\kappa}^{0} \pi^{+} ; \bar{\kappa}^{0} \rightarrow K_{S} \pi^{0}$ & $5.4 \times 10^{-3}$ & $5.4 \times 10^{-3}$ & $\left(6_{-4}^{+5}\right) \times 10^{-3}$ \\
\hline$D^{+} \rightarrow \bar{\kappa}^{0} K^{+} ; \bar{\kappa}^{0} \rightarrow K^{-} \pi^{+}$ & $3.7 \times 10^{-4}$ & $3.7 \times 10^{-4}$ & $\left(6.8_{-2.1}^{+3.5}\right) \times 10^{-4}$ \\
\hline$D^{0} \rightarrow f_{0} \pi^{0} ; f_{0} \rightarrow \pi^{+} \pi^{-}$ & $1.6 \times 10^{-5}$ & $1.4 \times 10^{-5}$ & $(3.7 \pm 0.9) \times 10^{-5}$ \\
\hline$D^{0} \rightarrow f_{0} \pi^{0} ; f_{0} \rightarrow K^{+} K^{-}$ & $1.1 \times 10^{-5}$ & $8.8 \times 10^{-6}$ & $(3.6 \pm 0.6) \times 10^{-4}$ \\
\hline$D^{0} \rightarrow f_{0} \bar{K}^{0} ; f_{0} \rightarrow \pi^{+} \pi^{-}$ & $9.0 \times 10^{-6}$ & $3.0 \times 10^{-4}$ & $\left(2.40_{-0.46}^{+0.80}\right) \times 10^{-3}$ \\
\hline$D^{0} \rightarrow f_{0} \bar{K}^{0} ; f_{0} \rightarrow K^{+} K^{-}$ & $4.3 \times 10^{-6}$ & $1.4 \times 10^{-4}$ & $<1.8 \times 10^{-4}$ \\
\hline$D^{0} \rightarrow a_{0}^{+} \pi^{-} ; a_{0}^{+} \rightarrow K^{+} \bar{K}^{0}$ & $1.3 \times 10^{-5}$ & $1.3 \times 10^{-5}$ & $(1.2 \pm 0.8) \times 10^{-3}$ \\
\hline$D^{0} \rightarrow a_{0}^{-} \pi^{+} ; a_{0}^{-} \rightarrow K^{-} K^{0}$ & $2.9 \times 10^{-4}$ & $2.9 \times 10^{-4}$ & $(2.6 \pm 2.8) \times 10^{-4}$ \\
\hline$D^{0} \rightarrow a_{0}^{+} K^{-} ; a_{0}^{+} \rightarrow K^{+} \bar{K}^{0}$ & $2.2 \times 10^{-4}$ & $2.2 \times 10^{-4}$ & $(1.47 \pm 0.33) \times 10^{-3}$ \\
\hline$D^{0} \rightarrow a_{0}^{0} \bar{K}^{0} ; a_{0}^{0} \rightarrow K^{+} K^{-}$ & $3.4 \times 10^{-4}$ & $3.4 \times 10^{-4}$ & $(6.18 \pm 0.73) \times 10^{-3}$ \\
\hline$D^{0} \rightarrow a_{0}^{0} \bar{K}^{0} ; a_{0}^{0} \rightarrow \eta \pi^{0}$ & $1.1 \times 10^{-3}$ & $1.1 \times 10^{-3}$ & $(2.40 \pm 0.56) \%$ \\
\hline$D^{0} \rightarrow a_{0}^{-} K^{+} ; a_{0}^{-} \rightarrow K^{-} \bar{K}^{0}$ & $1.7 \times 10^{-5}$ & $1.7 \times 10^{-5}$ & $<2.2 \times 10^{-4}$ \\
\hline$D^{0} \rightarrow \sigma \pi^{0} ; \sigma \rightarrow \pi^{+} \pi^{-}$ & $2.2 \times 10^{-5}$ & $2.0 \times 10^{-4}$ & $(1.22 \pm 0.22) \times 10^{-4}$ \\
\hline$D_{s}^{+} \rightarrow f_{0} \pi^{+} ; f_{0} \rightarrow K^{+} K^{-}$ & $2.5 \times 10^{-3}$ & $5.1 \times 10^{-3}$ & $(1.14 \pm 0.31) \%$ \\
\hline$D_{s}^{+} \rightarrow a_{0}^{+, 0} \pi^{0,+} ; a_{0}^{+, 0} \rightarrow \eta \pi^{+, 0}$ & 0 & 0 & $(1.46 \pm 0.27) \%$ \\
\hline$D^{+} \rightarrow a_{0}(1450)^{0} \pi^{+} ; a_{0}^{0} \rightarrow K^{+} K^{-}$ & & $1.7 \times 10^{-5}$ & $\left(4.5_{-1.8}^{+7.0}\right) \times 10^{-4}$ \\
\hline$D^{+} \rightarrow \bar{K}_{0}^{* 0} \pi^{+} ; \bar{K}_{0}^{* 0} \rightarrow K^{-} \pi^{+}$ & & $1.38 \%$ & $(1.25 \pm 0.06) \%$ \\
\hline$D^{+} \rightarrow \bar{K}_{0}^{* 0} \pi^{+} ; \bar{K}_{0}^{* 0} \rightarrow K_{S} \pi^{0}$ & & $6.0 \times 10^{-3}$ & $(5.4 \pm 1.8) \times 10^{-3}$ \\
\hline$D^{+} \rightarrow \bar{K}_{0}^{* 0} K^{+} ; \bar{K}_{0}^{* 0} \rightarrow K^{-} \pi^{+}$ & & $7.6 \times 10^{-5}$ & $(1.82 \pm 0.35) \times 10^{-3}$ \\
\hline$D^{0} \rightarrow a_{0}(1450)^{-} \pi^{+} ; a_{0}^{-} \rightarrow K^{-} K^{0}$ & & $6.1 \times 10^{-6}$ & $(5.0 \pm 4.0) \times 10^{-5}$ \\
\hline$D^{0} \rightarrow a_{0}(1450)^{+} \pi^{-} ; a_{0}^{+} \rightarrow K^{+} \bar{K}^{0}$ & & $1.8 \times 10^{-7}$ & $(6.4 \pm 5.0) \times 10^{-5}$ \\
\hline$D^{0} \rightarrow a_{0}(1450)^{-} K^{+} ; a_{0}^{-} \rightarrow K^{-} K_{S}$ & & & $<0.6 \times 10^{-3}$ \\
\hline$D^{0} \rightarrow K_{0}^{*-} \pi^{+} ; K_{0}^{*-} \rightarrow \bar{K}^{0} \pi^{-}$ & & $8.3 \times 10^{-4}$ & $\left(5.34_{-0.66}^{+0.80}\right) \times 10^{-3}$ \\
\hline$D^{0} \rightarrow K_{0}^{*-} \pi^{+} ; K_{0}^{*-} \rightarrow K^{-} \pi^{0}$ & & $4.2 \times 10^{-4}$ & $(4.8 \pm 2.2) \times 10^{-3}$ \\
\hline$D^{0} \rightarrow \bar{K}_{0}^{* 0} \pi^{0} ; \bar{K}_{0}^{* 0} \rightarrow K^{-} \pi^{+}$ & & $9.6 \times 10^{-4}$ & $\left(5.9_{-1.6}^{+5.0}\right) \times 10^{-3}$ \\
\hline$D^{0} \rightarrow K_{0}^{*+} \pi^{-} ; K_{0}^{*+} \rightarrow K^{0} \pi^{+}$ & & $5.4 \times 10^{-6}$ & $<2.8 \times 10^{-5}$ \\
\hline$D_{s}^{+} \rightarrow K_{0}^{* 0} \pi^{+} ; K_{0}^{* 0} \rightarrow K^{+} \pi^{-}$ & & $1.3 \times 10^{-4}$ & $(5.0 \pm 3.5) \times 10^{-4}$ \\
\hline$D_{s}^{+} \rightarrow \bar{K}_{0}^{* 0} K^{+} ; \bar{K}_{0}^{* 0} \rightarrow K^{-} \pi^{+}$ & & $2.0 \times 10^{-3}$ & $(1.7 \pm 0.3) \times 10^{-3}$ \\
\hline
\end{tabular}

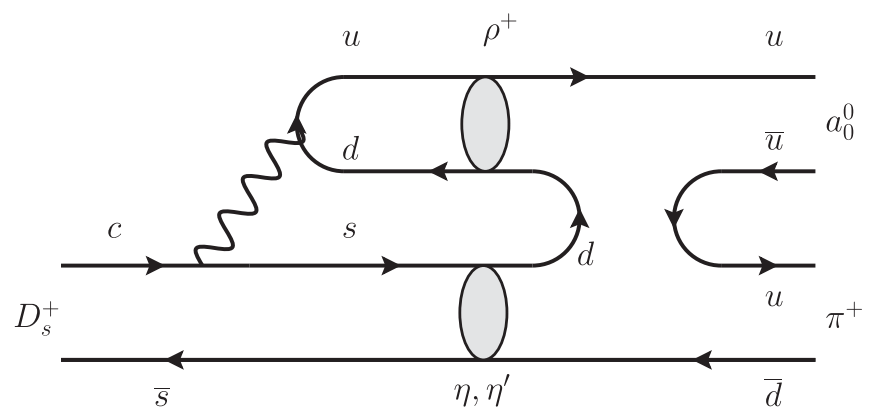

FIG. 1. Long-distance contributions to the $W$-annihilation amplitude of $D_{s}^{+} \rightarrow a_{0}^{0} \pi^{+}$through final-state rescattering of $\rho \eta^{\left({ }^{\prime}\right)} \rightarrow a_{0} \pi$. coupling constants for $\rho^{+} \rightarrow \pi^{+} \pi^{0}$ and $a_{0}^{0} \rightarrow \pi^{0} \eta$. The graph in Fig. 2(a) shows the resonant final-state interactions manifested by the nearby resonance $\pi(1800)$ whose strong decay to $a_{0} \pi$ has been seen experimentally [1]. However, we are not able to have a quantitative statement owing to the lack of information on its partial width.

Assuming $A^{\prime} \approx-A$, the annihilation amplitude extracted from the data of $D_{s}^{+} \rightarrow a_{0}^{+} \pi^{0}+a_{0}^{0} \pi^{+}$is (in units of $\left.10^{-6} \mathrm{GeV}\right)$,

$$
|A|=0.91 \pm 0.12
$$

Hence, the annihilation amplitude is very sizable in the $S P$ sector, $|A / T|_{S P} \sim 1 / 2$, contrary to its suppression 


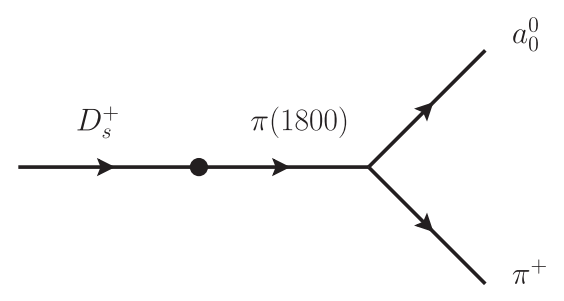

(a)

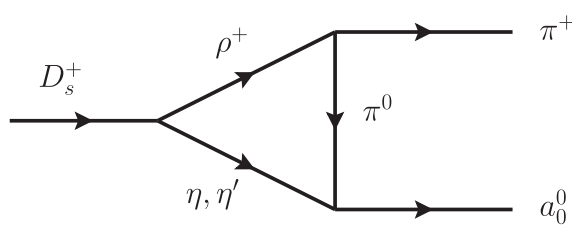

(b)

FIG. 2. Manifestation of Fig. 1 at the hadron level: (a) resonant contribution from the nearby resonance $\pi(1800)$ and (b) the triangle rescattering diagram.

$|A / T|_{P P} \sim 0.18$ in the $P P$ sector [80] and $\left|A_{V} / T_{P}\right|_{V P} \sim$ 0.07 in the $V P$ sector [81].

\section{B. Finite width effects}

The finite-width effect is accounted for by the quantity $\eta_{R}$ defined by $[82,83]$

$$
\begin{aligned}
\eta_{R} & \equiv \frac{\Gamma\left(D \rightarrow R P_{3} \rightarrow P_{1} P_{2} P_{3}\right)_{\Gamma_{R} \rightarrow 0}}{\Gamma\left(D \rightarrow R P_{3} \rightarrow P_{1} P_{2} P_{3}\right)} \\
& =\frac{\Gamma\left(D \rightarrow R P_{3}\right) \mathcal{B}\left(R \rightarrow P_{1} P_{2}\right)}{\Gamma\left(D \rightarrow R P_{3} \rightarrow P_{1} P_{2} P_{3}\right)}=1+\delta,
\end{aligned}
$$

so that the deviation of $\eta_{R}$ from unity measures the degree of departure from the NWA when the resonance width is finite. It is naïvely expected that the correction $\delta$ will be of order $\Gamma_{R} / m_{R}$. It is calculable theoretically but depends on the line shape of the resonance and the approach of describing weak hadronic decays such as QCD factorization and perturbative QCD.

Using the branching fractions of two-body and threebody $D$ decays calculated in Tables VII and VIII, respectively, in scheme II, the resultant $\eta_{R}$ parameters for scalar resonances $\sigma, \kappa$ and $K_{0}^{*}$ produced in the three-body $D$ decays are summarized in Table IX. We only consider the $D^{+}$decays as the three-body modes listed in Table IX are not contaminated by the $W$-annihilation amplitude and hence the calculated finite width effects are more trustworthy. We have also checked explicitly that $\eta_{R} \rightarrow 1$ in the narrow width limit as it should be. The $\eta_{R}$ parameters for various resonances produced in the three-body $B$ decays have been evaluated in $[82,83]$. Our results for $\eta_{R}$ 's in Table IX have similar features as the values $\eta_{\sigma / f_{0}(500)}=2.15 \pm 0.05$ and $\eta_{K_{0}^{*}(1430)}=0.83 \pm 0.04$ obtained in $B$ decays.

Note that a priori we do not know if the deviation of $\eta_{R}$ from unity is positive or negative. In general, it depends on the line shape, mass and width of the resonance. As alluded to above, the mass and width have a more dominant effect than the line shape in the case of $\kappa(700)$. As another example, we found in Ref. [83] that $\eta_{\rho}>1$ for the BreitWigner line shape and $\eta_{\rho}<1$ when the Gounaris-Sakurai model [84] is used to describe the line shape of the broad $\rho(770)$ resonance. To our knowledge, there is no good argument favoring one line shape over the other. Therefore, $\eta_{K_{0}^{*}(1430)}=0.985<1$, for example, is the result of our particular line shape choice.

When the resonance is sufficiently broad, it is necessary to take into account the finite-width effects characterized by the parameter $\eta_{R}$. Explicitly [82,83],

$$
\begin{aligned}
\mathcal{B}(D \rightarrow R P) & =\eta_{R} \mathcal{B}(D \rightarrow R P)_{\mathrm{NWA}} \\
& =\eta_{R} \frac{\mathcal{B}\left(D \rightarrow R P_{3} \rightarrow P_{1} P_{2} P_{3}\right)_{\mathrm{expt}}}{\mathcal{B}\left(R \rightarrow P_{1} P_{2}\right)_{\text {expt }}}
\end{aligned}
$$

Therefore, the experimental branching fractions $\mathcal{B}(D \rightarrow$ $R P)_{\text {NWA }}$ for $D^{+} \rightarrow \sigma \pi^{+}, \bar{\kappa}^{0} \pi^{+}$and $\bar{K}_{0}^{* 0} \pi^{+}$decays in Tables I and VII should have the following corrections:

$$
\begin{aligned}
& \mathcal{B}\left(D^{+} \rightarrow \sigma \pi^{+}\right):(2.1 \pm 0.2) \times 10^{-3} \rightarrow(3.8 \pm 0.3) \times 10^{-3}, \\
& \mathcal{B}\left(D^{+} \rightarrow \bar{\kappa}^{0} \pi^{+}\right):\left(3.6_{-2.4}^{+3.0}\right) \% \rightarrow\left(6.7_{-4.5}^{+5.6}\right) \%, \\
& \mathcal{B}\left(D^{+} \rightarrow \bar{K}_{0}^{* 0} \pi^{+}\right):(1.98 \pm 0.22) \% \rightarrow(1.94 \pm 0.22) \% .
\end{aligned}
$$

TABLE IX. A summary of the $\eta_{R}$ parameter for scalar resonances produced in the three-body $D$ decays. The mass and width of $\sigma / f_{0}(500)$ are taken from Eq. (5.23).

\begin{tabular}{lcccc}
\hline \hline Resonance & $D \rightarrow R h_{3} \rightarrow h_{1} h_{2} h_{3}$ & $\Gamma_{R}(\mathrm{MeV})[1]$ & $m_{R}(\mathrm{MeV})[1]$ & $\Gamma_{R} / m_{R}$ \\
\hline$\sigma / f_{0}(500)$ & $D^{+} \rightarrow \sigma \pi^{+} \rightarrow \pi^{+} \pi^{-} \pi^{+}$ & $700 \pm 26$ & $563 \pm 10$ & $1.243 \pm 0.051$ \\
$\kappa / K_{0}^{*}(700)$ & $D^{+} \rightarrow \bar{\kappa}^{0} \pi^{+} \rightarrow K_{S}^{0} \pi^{0} \pi^{+}$ & $468 \pm 30$ & $845 \pm 17$ & 1.850 \\
$K_{0}^{*}(1430)$ & $D^{+} \rightarrow \bar{K}_{0}^{* 0} \pi^{+} \rightarrow K^{-} \pi^{+} \pi^{+}$ & $270 \pm 80$ & $1425 \pm 50$ & $0.554 \pm 0.037$ \\
\hline \hline
\end{tabular}


From Table VII, it is evident that the agreement between theory and experiment is substantially improved for $D^{+} \rightarrow \sigma \pi^{+}$and $D^{+} \rightarrow \bar{\kappa}^{0} \pi^{+}$.

If we employ the pole mass and width, $m_{\kappa}=648 \pm$ $7 \mathrm{MeV}$ and $\Gamma_{\kappa}=560 \pm 32 \mathrm{MeV}$, respectively, for $\kappa / K_{0}^{*}(700)$ and the pole line shape given in Eq. (5.24), we will be led to the results $\mathcal{B}\left(D^{+} \rightarrow \bar{\kappa}^{0} \pi^{+}\right)=8.10 \%$, $\mathcal{B}\left(D^{+} \rightarrow \bar{\kappa}^{0} \pi^{+} \rightarrow K_{S}^{0} \pi^{0} \pi^{+}\right)=1.62 \times 10^{-3}$ and $\eta_{\kappa}=8.34$. This implies that the finite-width correction will be unreasonably too large and thus unlikely, as alluded to at the end of Sec. V E. However, if the Breit-Wigner mass and width are used instead, we get $\eta_{\kappa}=1.92$ for pole line shape, which is a more reasonable result. This implies that in this case, it is the mass and width rather than the line shape that governs the finite-width correction.

For the case of $f_{0}(500)$, one may wonder what the correction will be if the Breit-Wigner line shape is used. According to PDG [1], the Breit-Wigner mass and width of $f_{0}(500)$ lie in the wide ranges of $400-800 \mathrm{MeV}$ and 100 $800 \mathrm{MeV}$, respectively. As a result, it is quite difficult to pin down a specific set of parameters and thereby determine the finite-width correction. On the contrary, LHCb has determined its pole mass and width with reasonable accuracy using the pole line shape [see Eq. (5.23)]. It turns out that the pole mass and width fall within the above allowed ranges of the Breit-Wigner mass and width. Therefore, it is more sensible to use pole mass and width for calculations in either line shapes.

\section{CONCLUSIONS}

In this work we have examined the quasi-two-body $D \rightarrow S P$ decays and the three-body $D$ decays proceeding through intermediate scalar resonances. Our main results are the following:

(i) In the $D \rightarrow S P_{3} \rightarrow P_{1} P_{2} P_{3}$ decays, we cannot extract the two-body branching fractions $\mathcal{B}(D \rightarrow S P)$ for $S=f_{0}(980)$ and $a_{0}(980)$ due to the lack of information of $\mathcal{B}\left(S \rightarrow P_{1} P_{2}\right)$ [except for $a_{0}(980) \rightarrow \pi \eta$ ]. For $S=\kappa / K_{0}^{*}(700)$ and $\sigma / f_{0}(500)$, the extracted two-body branching fractions are subject to large finite-width effects owing to their broad widths. Hence, for light scalars it is more sensible to study $\mathcal{B}\left(D \rightarrow S P \rightarrow P_{1} P_{2} P\right)$ directly and compare with experiment.

(ii) We have considered the two-quark (scheme I) and four-quark (scheme II) descriptions of the light scalar mesons with masses below or close to $1 \mathrm{GeV}$. Recent BESIII measurements of semileptonic charm decays favor the $\mathrm{SU}(3)$ nonet tetraquark description of the $f_{0}(500), f_{0}(980)$ and $a_{0}(980)$ produced in charmed meson decay. In Table VIII we have calculated $D \rightarrow S P_{3} \rightarrow P_{1} P_{2} P_{3}$ in schemes I and II. It is evident that scheme II agrees better with experiment for decays such as $D^{+} \rightarrow f_{0} \pi^{+}$followed by $f_{0} \rightarrow \pi^{+} \pi^{-}$and $D^{+} \rightarrow f_{0} K^{+}$followed by $f_{0} \rightarrow$ $\pi^{+} \pi^{-}$or $f_{0} \rightarrow K^{+} K^{-}$. This again favors the tetraquark structure for light scalars. The predicted rates for $D^{0} \rightarrow f_{0} P, a_{0} P$ are generally smaller than experimental data by one order of magnitude, presumably implying the importance of $W$-exchange.

(iii) The three-body decay modes $D^{+} \rightarrow \bar{\kappa}^{0}\left(\rightarrow K_{S} \pi^{0}\right) \pi^{+}$, $D^{+} \rightarrow \bar{K}_{0}^{*}\left(\rightarrow K^{-} \pi^{+}\right) \pi^{+}$and $D^{+} \rightarrow \bar{K}_{0}^{*}\left(\rightarrow K_{S} \pi^{0}\right) \pi^{+}$ are ideal for testing the validity of the factorization approach as they are free of $W$-annihilation contributions. $T$ and $C^{\prime}$ amplitudes contribute constructively, contrary to the Cabibbo-allowed $D^{+} \rightarrow \bar{K}^{0} \pi^{+}$ decay where the interference between external and internal $W$-emission is destructive.

(iv) Denoting the primed amplitudes $T^{\prime}$ and $C^{\prime}$ for the case when the emitted meson is a scalar meson, it is naïvely expected that $T^{\prime}=C^{\prime}=0$ for the neutral scalars $\sigma, f_{0}$ and $a_{0}^{0},\left|T^{\prime}\right| \ll|T|$ and $\left|C^{\prime}\right| \ll|C|$ for the charged $a_{0}$ and $\left|T^{\prime}\right|<|T|$ and $\left|C^{\prime}\right|<|C|$ for the $\kappa$ and $K_{0}^{*}(1430)$. Beyond the factorization approximation, contributions proportional to the scalar decay constant $\bar{f}_{S}$ can be produced from vertex and hard spectator-scattering corrections for the abovementioned neutral scalars.

(v) We have studied the flavor operators $a_{1,2}\left(M_{1} M_{2}\right)$ for $M_{1} M_{2}=S P$ and $P S$ within the framework of QCD factorization. Notice that $a_{i}(P S)$ and $a_{i}(S P)$ are very different as the former does not receive factorizable contributions. While $a_{1,2}(S P)$ are similar for any light and heavy scalar mesons, $a_{1}(P S)$ and $a_{2}(P S)$ vary from neutral to the charged ones as shown in Table VI. The flavor operators $a_{1,2}\left(\pi a_{0}^{ \pm}\right)$ are much greater than $a_{1,2}\left(\pi a_{0}^{0}\right)$. In general, $a_{1,2}(P S)$ become larger when the vector decay constants become smaller.

(vi) For $f_{0}(980)$ and $a_{0}(980)$, we use the Flatté line shape to describe both of them to take into account the threshold and coupled channel effects. For the very broad $\sigma / f_{0}(500)$, we follow LHCb to employ a simple pole description.

(vii) The annihilation amplitude inferred from the measurement of $D_{s}^{+} \rightarrow a_{0}^{+, 0} \pi^{0,+} \rightarrow \eta \pi^{+, 0} \pi^{0,+}$ is given by $|A|=(0.91 \pm 0.12) \times 10^{-6} \mathrm{GeV}$. It is very sizable in the $S P$ sector, $|A / T|_{S P} \sim 1 / 2$, contrary to its suppression in the $P P$ sector with $|A / T|_{P P} \sim 0.18$.

(viii) Since $\sigma$ and $\kappa$ are very broad, we have considered their finite-width effects characterized by the parameter $\eta_{S}$, whose deviation from unity measures the degree of departure from the NWA when the resonance width is finite. We find $\eta_{\sigma}$ and $\eta_{\kappa}$ to be of order 1.85-1.87. The experimental branching fractions $\mathcal{B}\left(D^{+} \rightarrow \sigma \pi^{+}\right)$and $\mathcal{B}\left(D^{+} \rightarrow \bar{\kappa}^{0} \pi^{+}\right)$should then $\operatorname{read}(3.8 \pm 0.3) \times 10^{-3}$ and $\left(6.7_{-4.5}^{+5.6}\right) \%$, respectively. 
(ix) For each scalar nonet (lighter and heavier one) we have 15 unknown parameters for the 8 topological amplitudes $T, C, E, A$ and $T^{\prime}, C^{\prime}, E^{\prime}, A^{\prime}$. However, there are only 14 independent data to fit. Moreover, since we need to introduce appropriate energydependent line shapes for the scalar mesons, it is not conceivable to extract the topological amplitudes from three-body decays as the decay rates cannot be factorized into the topological amplitude squared and the phase space factor.

\section{ACKNOWLEDGMENTS}

This research was supported in part by the Ministry of Science and Technology of R.O.C. under Grants No. MOST-107-2119-M-001-034, No. MOST-110-2112M-001-025 and No. MOST-108-2112-M-002-005-MY3, the National Natural Science Foundation of China under Grant No. 11347030, and the Program of Science and Technology Innovation Talents in Universities of Henan Province 14HASTIT037.
[1] P. A. Zyla et al. (Particle Data Group), Review of particle physics, Prog. Theor. Exp. Phys. 2020, 083 C01 (2020) and 2021 update.

[2] R. Aaij et al. (LHCb Collaboration), Dalitz plot analysis of the $D^{+} \rightarrow K^{-} K^{+} K^{+}$decay, J. High Energy Phys. 04 (2019) 063.

[3] M. Ablikim et al. (BESIII Collaboration), Amplitude analysis and branching fraction measurement of the decay $D_{s}^{+} \rightarrow \pi^{+} \pi^{0} \pi^{0}$, J. High Energy Phys. 01 (2022) 052 .

[4] M. Ablikim et al. (BESIII Collaboration), Amplitude analysis and branching fraction measurement of $D_{s}^{+} \rightarrow$ $K^{+} K^{-} \pi^{+}$, Phys. Rev. D 104, 012016 (2021).

[5] M. Ablikim et al. (BESIII Collaboration), Amplitude Analysis of $D_{s}^{+} \rightarrow \pi^{+} \pi^{0} \eta$ and First Observation of the Pure $W$-Annihilation Decays $D_{s}^{+} \rightarrow a_{0}(980)^{+} \pi^{0}$ and $D_{s}^{+} \rightarrow a_{0}(980)^{0} \pi^{+}$, Phys. Rev. Lett. 123, 112001 (2019).

[6] M. Ablikim et al. (BESIII Collaboration), Analysis of the decay $D^{0} \rightarrow K_{S}^{0} K^{+} K^{-}$, arXiv:2006.02800.

[7] F. Hussain, A. N. Kamal, and S. N. Sinha, Cabibbo angle favored decays: $D \rightarrow S P$, Z. Phys. C 36, 199 (1987).

[8] A. C. Katoch and R. C. Verma, D decays into pseudoscalar and scalar mesons, Z. Phys. C 62, 173 (1994).

[9] S. Fajfer, $D \rightarrow P S$ decays and the effective chiral Lagrangian for heavy and light mesons, Z. Phys. C 68, 81 (1995).

[10] F. Buccella, M. Lusignoli, and A. Pugliese, Charm nonleptonic decays and final state interactions, Phys. Lett. B 379, 249 (1996).

[11] H. Y. Cheng, Hadronic $D$ decays involving scalar mesons, Phys. Rev. D 67, 034024 (2003).

[12] B. El-Bennich, O. Leitner, J. P. Dedonder, and B. Loiseau, Scalar meson $f_{0}(980)$ in heavy-meson decays, Phys. Rev. D 79, 076004 (2009).

[13] D. R. Boito, J. P. Dedonder, B. El-Bennich, O. Leitner, and B. Loiseau, Scalar resonances in a unitary $\pi-\pi S$-wave model for $D^{+} \rightarrow \pi^{+} \pi^{-} \pi^{+}$, Phys. Rev. D 79, 034020 (2009).

[14] H. Y. Cheng and C. W. Chiang, Hadronic D decays involving even-parity light mesons, Phys. Rev. D 81, 074031 (2010).

[15] J. P. Dedonder, R. Kaminski, L. Lesniak, and B. Loiseau, Dalitz plot studies of $D^{0} \rightarrow K_{S}^{0} \pi^{+} \pi^{-}$decays in a factorization approach, Phys. Rev. D 89, 094018 (2014).
[16] J. J. Xie, L. R. Dai, and E. Oset, The low lying scalar resonances in the $D^{0}$ decays into $K_{s}^{0}$ and $f_{0}(500), f_{0}(980)$, $a_{0}(980)$, Phys. Lett. B 742, 363 (2015).

[17] B. Loiseau, Theory overview on amplitude analyses with charm decays, Proc. Sci., CHARM2016 (2016) 033 [arXiv:1611.05286].

[18] J. P. Dedonder, R. Kamiński, L. Leśniak, and B. Loiseau, Dalitz plot studies of $D^{0} \rightarrow K_{S}^{0} K^{+} K^{-}$decays in a factorization approach, Phys. Rev. D 103, 114028 (2021).

[19] C. Amsler and N. A. Tornqvist, Mesons beyond the naïve quark model, Phys. Rep. 389, 61 (2004).

[20] F. E. Close and N. A. Tornqvist, Scalar mesons above and below $1 \mathrm{GeV}$, J. Phys. G 28, R249 (2002).

[21] T. Barnes, Two photon decays support the $(K \bar{K})$ molecule picture of the $S^{*}(975)$ and $\delta(980)$, Phys. Lett. 165B, 434 (1985).

[22] N. N. Achasov and V. N. Ivanchenko, On a search for four quark states in radiative decays of $\phi$ meson, Nucl. Phys. B315, 465 (1989).

[23] N. N. Achasov, Radiative decays of $\phi$ meson about nature of light scalar resonances, Nucl. Phys. A728, 425 (2003).

[24] D. Black, M. Harada, and J. Schechter, Chiral approach to $\phi$ radiative decays, Phys. Rev. D 73, 054017 (2006).

[25] R. L. Jaffe, Multiquark hadrons. I. Phenomenology of $Q^{2} \bar{Q}^{2}$ mesons, Phys. Rev. D 15, 267 (1977); Multiquark hadrons. II. Methods, Phys. Rev. D 15, 281 (1977).

[26] Y. Kuroda, M. Harada, S. Matsuzaki, and D. Jido, Inverse mass hierarchy of light scalar mesons driven by anomalyinduced flavor breaking, Prog. Theor. Exp. Phys. 2020, 053D02 (2020).

[27] S. Prelovsek, T. Draper, C. B. Lang, M. Limmer, K. F. Liu, N. Mathur, and D. Mohler, Lattice study of light scalar tetraquarks with $I=0,2,1 / 2,3 / 2$ : Are $\sigma$ and $\kappa$ tetraquarks?, Phys. Rev. D 82, 094507 (2010); S. Prelovsek and D. Mohler, A lattice study of light scalar tetraquarks, Phys. Rev. D 79, 014503 (2009).

[28] N. Mathur, A. Alexandru, Y. Chen, S. J. Dong, T. Draper, I. Horvath, F. X. Lee, K. F. Liu, S. Tamhankar, and J. B. Zhang, Scalar mesons $a_{0}(1450)$ and $\sigma(600)$ from lattice QCD, Phys. Rev. D 76, 114505 (2007).

[29] C. Alexandrou, J. O. Daldrop, M. Dalla Brida, M. Gravina, L. Scorzato, C. Urbach, and M. Wagner, Lattice investigation 
of the scalar mesons $a_{0}(980)$ and $\kappa$ using four-quark operators, J. High Energy Phys. 04 (2013) 137.

[30] C. Alexandrou, J. Berlin, M. Dalla Brida, J. Finkenrath, T. Leontiou, and M. Wagner, Lattice QCD investigation of the structure of the $a_{0}$ (980) meson, Phys. Rev. D97, 034506 (2018).

[31] M. Wakayama, T. Kunihiro, S. Muroya, A. Nakamura, C. Nonaka, M. Sekiguchi, and H. Wada, Lattice QCD study of four-quark components of the isosinglet scalar mesons: Significance of disconnected diagrams, Phys. Rev. D 91, 094508 (2015).

[32] J. A. Oller, E. Oset, and J. R. Pelaez, Nonperturbative Approach to Effective Chiral Lagrangians and Meson Interactions, Phys. Rev. Lett. 80, 3452 (1998).

[33] J. A. Oller, E. Oset, and J. R. Pelaez, Meson meson interaction in a nonperturbative chiral approach, Phys. Rev. D 59, 074001 (1999); 60, 099906(E) (1999); 75, 099903(E) (2007).

[34] J. D. Weinstein and N. Isgur, $K \bar{K}$ molecules, Phys. Rev. D 41, 2236 (1990).

[35] H. Y. Cheng, C. K. Chua, and K. C. Yang, Charmless hadronic $\mathrm{B}$ decays involving scalar mesons: Implications to the nature of light scalar mesons, Phys. Rev. D 73, 014017 (2006).

[36] R. Fleischer, R. Knegjens, and G. Ricciardi, Anatomy of $B_{s, d}^{0} \rightarrow J / \psi f_{0}(980)$, Eur. Phys. J. C 71, 1832 (2011).

[37] R. Aaij et al. (LHCb Collaboration), Analysis of the resonant components in $B^{0} \rightarrow J / \psi \pi^{+} \pi^{-}$, Phys. Rev. D 87, 052001 (2013).

[38] L. Maiani, F. Piccinini, A. D. Polosa, and V. Riquer, New Look at Scalar Mesons, Phys. Rev. Lett. 93, 212002 (2004).

[39] W. Wang and C. D. Lu, Distinguishing two kinds of scalar mesons from heavy meson decays, Phys. Rev. D 82, 034016 (2010).

[40] M. Ablikim et al. (BESIII Collaboration), Observation of the Semileptonic Decay $D^{0} \rightarrow a_{0}(980)^{-} e^{+} \nu_{e}$ and Evidence for $D^{+} \rightarrow a_{0}(980)^{0} e^{+} \nu_{e}$, Phys. Rev. Lett. 121, 081802 (2018).

[41] M. Ablikim et al. (BESIII Collaboration), Observation of $D^{+} \rightarrow f_{0}(500) e^{+} \nu_{e}$ and Improved Measurements of $D \rightarrow \rho e^{+} \nu_{e}$, Phys. Rev. Lett. 122, 062001 (2019).

[42] N. N. Achasov, A. V. Kiselev, and G. N. Shestakov, Semileptonic decays $D \rightarrow \pi^{+} \pi^{-} e^{+} \nu_{e}$ and $D_{s} \rightarrow \pi^{+} \pi^{-} e^{+} \nu_{e}$ as the probe of constituent quark-antiquark pairs in the light scalar mesons, Phys. Rev. D 102, 016022 (2020).

[43] M. Wirbel, B. Stech, and M. Bauer, Exclusive semileptonic decays of heavy mesons, Z. Phys. C 29, 637 (1985); M. Bauer, B. Stech, and M. Wirbel, Exclusive nonleptonic decays of $D, D_{s}$, and $B$ mesons, Z. Phys. C 34, 103 (1987).

[44] H. Y. Cheng, C. K. Chua, and C. W. Hwang, Covariant light-front approach for s-wave and p-wave mesons: Its application to decay constants and form factors, Phys. Rev. D 69, 074025 (2004).

[45] R. C. Verma, Decay constants and form factors of s-wave and p-wave mesons in the covariant light-front quark model, J. Phys. G 39, 025005 (2012).

[46] N. R. Soni, A. N. Gadaria, J. J. Patel, and J. N. Pandya, Semileptonic decays of charmed mesons to light scalar mesons, Phys. Rev. D 102, 016013 (2020).
[47] Y. J. Shi, W. Wang, and S. Zhao, Chiral dynamics, S-wave contributions and angular analysis in $D \rightarrow \pi \pi \ell \bar{\nu}$, Eur. Phys. J. C 77, 452 (2017).

[48] X. D. Cheng, H. B. Li, B. Wei, Y. G. Xu, and M. Z. Yang, Study of $D \rightarrow a_{0}(980) e^{+} \nu_{e}$ decay in the light-cone sum rules approach, Phys. Rev. D 96, 033002 (2017).

[49] Q. Huang, Y. J. Sun, D. Gao, G. H. Zhao, B. Wang, and W. Hong, Study of form factors and branching ratios for $D \rightarrow S, A l \bar{\nu}_{l}$ with light-cone sum rules, arXiv:2102.12241.

[50] M. Ablikim et al. (BESIII Collaboration), Observation of the Semileptonic Decay $D^{0} \rightarrow a_{0}(980)^{-} e^{+} \nu_{e}$ and Evidence for $D^{+} \rightarrow a_{0}(980)^{0} e^{+} \nu_{e}$, Phys. Rev. Lett. 121, 081802 (2018).

[51] H. Y. Cheng and X. W. Kang, Branching fractions of semileptonic $D$ and $D_{s}$ decays from the covariant lightfront quark model, Eur. Phys. J. C 77, 587 (2017); 77, 863 (E) (2017).

[52] L. L. Chau and H. Y. Cheng, Analysis of exclusive twobody decays of charm mesons using the quark diagram scheme, Phys. Rev. D 36, 137 (1987); Analysis of the recent data of exclusive two-body charm decays, Phys. Lett. B 222, 285 (1989).

[53] L. L. Chau, Quark mixing in weak interactions, Phys. Rep. 95, 1 (1983).

[54] L. L. Chau and H. Y. Cheng, Quark Diagram Analysis of Two-body Charm Decays, Phys. Rev. Lett. 56, 1655 (1986).

[55] H. Y. Cheng, C. K. Chua, and K. C. Yang, Charmless hadronic $B$ decays involving scalar mesons: Implications to the nature of light scalar mesons, Phys. Rev. D 73, 014017 (2006).

[56] H. Y. Cheng, C. K. Chua, K. C. Yang, and Z. Q. Zhang, Revisiting charmless hadronic $B$ decays to scalar mesons, Phys. Rev. D 87, 114001 (2013).

[57] H. Y. Cheng and C. W. Chiang, Two-body hadronic charmed meson decays, Phys. Rev. D 81, 074021 (2010).

[58] B. Bhattacharya and J. L. Rosner, Flavor symmetry and decays of charmed mesons to pairs of light pseudoscalars, Phys. Rev. D 77, 114020 (2008).

[59] B. Bhattacharya and J. L. Rosner, Decays of charmed mesons to PV final states, Phys. Rev. D 79, 034016 (2009).

[60] B. Bhattacharya and J. L. Rosner, Charmed meson decays to two pseudoscalars, Phys. Rev. D 81, 014026 (2010).

[61] H. Y. Cheng and C. W. Chiang, Direct $C P$ violation in twobody hadronic charmed meson decays, Phys. Rev. D 85, 034036 (2012); 85, 079903(E) (2012).

[62] H. Y. Cheng and C. W. Chiang, SU(3) symmetry breaking and $C P$ violation in $D \rightarrow P P$ decays, Phys. Rev. D 86, 014014 (2012).

[63] H.-N. Li, C. D. Lu, and F. S. Yu, Branching ratios and direct $C P$ asymmetries in $D \rightarrow P P$ decays, Phys. Rev. D 86, 036012 (2012).

[64] Q. Qin, H. N. Li, C. D. Lü, and F. S. Yu, Branching ratios and direct $C P$ asymmetries in $D \rightarrow P V$ decays, Phys. Rev. D 89, 054006 (2014).

[65] H. Y. Cheng, C. W. Chiang, and A. L. Kuo, Global analysis of two-body $D \rightarrow V P$ decays within the framework of flavor symmetry, Phys. Rev. D 93, 114010 (2016).

[66] H. Y. Cheng and C. W. Chiang, $C P$ violation in quasi-twobody $D \rightarrow V P$ decays and three-body $D$ decays mediated by vector resonances, Phys. Rev. D 104, 073003 (2021). 
[67] M. Beneke, G. Buchalla, M. Neubert, and C. T. Sachrajda, QCD Factorization for $B \rightarrow \pi \pi$ Decays: Strong Phases and $C P$ Violation in the Heavy Quark Limit, Phys. Rev. Lett. 83, 1914 (1999); QCD factorization for exclusive, non-leptonic B meson decays: General arguments and the case of heavylight final states, Nucl. Phys. B591, 313 (2000).

[68] M. Beneke and M. Neubert, QCD factorization for $B \rightarrow P P$ and $B \rightarrow P V$ decays, Nucl. Phys. B675, 333 (2003).

[69] M. A. Ivanov, J. G. Körner, J. N. Pandya, P. Santorelli, N. R. Soni, and C. T. Tran, Exclusive semileptonic decays of D and $\mathrm{D}_{s}$ mesons in the covariant confining quark model, Front. Phys. (Beijing) 14, 64401 (2019).

[70] S. M. Flatte, Coupled-channel analysis of the $\pi \eta$ and $K \bar{K}$ systems near $K \bar{K}$ threshold, Phys. Lett. 63B, 224 (1976).

[71] F. Ambrosino et al. (KLOE Collaboration), Dalitz plot analysis of $e^{+} e^{-} \rightarrow \pi^{0} \pi^{0} \gamma$ events at $\sqrt{s}$ approximately $M(\phi)$ with the KLOE detector, Eur. Phys. J. C 49, 473 (2007).

[72] M. Ablikim et al. (BESIII Collaboration), Amplitude analysis of the $\chi_{c 1} \rightarrow \eta \pi^{+} \pi^{-}$decays, Phys. Rev. D 95, 032002 (2017).

[73] T. Mori et al. (Belle Collaboration), High statistics study of $f_{0}(980)$ resonance in $\gamma \gamma \rightarrow \pi^{+} \pi^{-}$production, Phys. Rev. D 75, 051101 (2007).

[74] J. R. Pelaez, From controversy to precision on the sigma meson: A review on the status of the non-ordinary $f_{0}(500)$ resonance, Phys. Rep. 658, 1 (2016).

[75] R. Aaij et al. (LHCb Collaboration), Amplitude analysis of the $B^{+} \rightarrow \pi^{+} \pi^{+} \pi^{-}$decay, Phys. Rev. D 101, 012006 (2020).
[76] J. R. Peláez and A. Rodas, Determination of the Lightest Strange Resonance $K_{0}^{*}(700)$ or $\kappa$, from a Dispersive Data Analysis, Phys. Rev. Lett. 124, 172001 (2020).

[77] H. Y. Cheng, C. K. Chua, and A. Soni, Final state interactions in hadronic $B$ decays, Phys. Rev. D 71, 014030 (2005).

[78] Y. K. Hsiao, Y. Yu, and B. C. Ke, Resonant $a_{0}(980)$ state in triangle rescattering $D_{s}^{+} \rightarrow \pi^{+} \pi^{0} \eta$ decays, Eur. Phys. J. C 80, 895 (2020).

[79] X. Z. Ling, M. Z. Liu, J. X. Lu, L. S. Geng, and J. J. Xie, Can the nature of $a_{0}(980)$ be tested in the $D_{s}^{+} \rightarrow \pi^{+} \pi^{0} \eta$ decay?, Phys. Rev. D 103, 116016 (2021).

[80] H. Y. Cheng and C. W. Chiang, Revisiting $C P$ violation in $D \rightarrow P P$ and $V P$ decays, Phys. Rev. D 100, 093002 (2019).

[81] H. Y. Cheng and C. W. Chiang, $C P$ violation in quasi-twobody $D \rightarrow V P$ decays and three-body $D$ decays mediated by vector resonances, Phys. Rev. D 104, 073003 (2021).

[82] H. Y. Cheng, C. W. Chiang, and C. K. Chua, Width effects in resonant three-body decays: $B$ decay as an example, Phys. Lett. B 813, 136058 (2021).

[83] H. Y. Cheng, C. W. Chiang, and C. K. Chua, Finite-width effects in three-body $B$ decays, Phys. Rev. D 103, 036017 (2021).

[84] G. J. Gounaris and J. J. Sakurai, Finite Width Corrections to the Vector Meson Dominance Prediction for $\rho \rightarrow e^{+} e^{-}$, Phys. Rev. Lett. 21, 244 (1968). 